\title{
Classical Fields Near Thermal Equilibrium
}

\author{
Carsten Greiner ${ }^{1}$ and Berndt Müller ${ }^{2}$ \\ ${ }^{1}$ Institut für Theoretische Physik, Justus-Liebig-Universität Giessen, \\ D-35392 Giessen, Germany, \\ ${ }^{2}$ Department of Physics, Duke University, \\ Durham, North Carolina, 27708-0305, USA.
}

\begin{abstract}
We discuss the classical limit for the long-distance ("soft") modes of a quantum field when the hard modes of the field are in thermal equilibrium. We address the question of the correct semiclassical dynamics when a momentum cut-off $|\mathbf{p}| \leq k_{c} \ll T$ is introduced. Higher order contributions leads to a stochastic interpretation for the effective action in analogy to Quantum Brownian Motion, resulting in dissipation and decoherence for the evolution of the soft modes. Particular emphasis is put on the understanding of dissipation. Our discussion focuses mostly on scalar fields, but we make some remarks on the extension to gauge theories.
\end{abstract}

\section{INTRODUCTION}

Solutions of the classical field equations in Minkowski space have been widely used in recent years to describe longdistance properties of quantum fields that require a nonperturbative analysis. These applications include: the diffusion rate of the topological charge in the electroweak gauge theory [1 5 , the thermalization rate of non-Abelian gauge fields [6 8], as well as a wide range of cosmological problems, such as thermalization, decoherence, and structure formation [9 16]. Many of these studies are concerned with dynamical properties of quantum fields near thermal equilibrium.

Classical treatments of the long-distance dynamics of bosonic quantum fields at high temperature are based on the observation that the average thermal amplitude of low-momentum modes is large. For a weakly coupled quantum field the occupation number of a mode with wave vector $\mathbf{p}$ and frequency $\omega_{\mathbf{p}}$ is given by the Bose distribution

$$
n\left(\omega_{\mathbf{p}}\right)=\left(e^{\hbar \omega_{\mathbf{p}} / T}-1\right)^{-1} .
$$

For temperatures $T$ much higher than the (dynamical) mass scale $m^{*}$ of the quantum field, the occupation number becomes large and approaches the classical equipartition limit

$$
n\left(\omega_{\mathbf{p}}\right) \stackrel{|\mathbf{p}| \rightarrow 0}{\longrightarrow} \frac{T}{m^{*}} \gg 1 .
$$

The classical field equations should provide a good approximation for the dynamics of such highly occupied modes.7.

At a closer look, however, the cogency of this heuristic argument suffers considerably. The thermodynamics of a classical field 7 is only defined if an ultraviolet cut-off $k_{c}$ is imposed on the momentum $\mathbf{p}$ such as a finite lattice spacing $a$. Many, if not most, thermodynamical properties of the classical field depend strongly on the value of the cut-off parameter $k_{c}$ and diverge in the continuum limit $\left(k_{c} \rightarrow \infty\right)$. Examples are the energy density

$$
\epsilon(T) \sim \int \frac{d^{3} p}{(2 \pi \hbar)^{3}} \hbar \omega_{\mathbf{p}} n\left(\omega_{\mathbf{p}}\right) \sim T k_{c}^{3}
$$

and the dynamical mass gap

\footnotetext{
${ }^{1}$ Note that for interacting quantum fields, generally, the dynamical mass gap at finite temperature is of order $g T$, where $g$ is a dimensionless coupling constant. Hence, the validity of (2) requires weak coupling. On the other hand, rapid thermalization and decoherence of the soft modes requires that the coupling to the heat bath is sufficiently strong [17]. It needs to be established whether both conditions can be satisfied simultaneously.

${ }^{2}$ Indeed, the ultraviolet divergence of thermodynamic quantities was one of the motivations for Planck's conjecture in 1900 that the excitations of field modes are quantized.
} 


$$
m^{* 2} \sim \frac{g^{2}}{2} \int \frac{d^{3} p}{(2 \pi)^{3}} \frac{1}{\omega_{\mathbf{p}}} n\left(\omega_{\mathbf{p}}\right) \sim \frac{g^{2}}{2} T \hbar k_{c}
$$

in the high-temperature limit (and for $k_{c} \gg m^{*}$ ), which contrasts with the results $\epsilon(T) \sim T^{4}$ and $m^{* 2} \sim g^{2} T^{2}$ in the full quantum theory. Remarkably, some thermodynamic quantities, such as the diffusion rate of the topological charge [2, [] in non-Abelian gauge theories or the maximal Lyapunov exponent [6], which is equivalent to the damping rate of soft thermal excitations [8], are found to be insensitive to the cut-off, attaining a finite value in the continuum limit.

The question arises whether the value reached in the continuum limit agrees with the one obtained in the full thermal quantum field theory, if a complete nonperturbative calculation were feasible. Naively, one expects that any quantity that is independent of $\hbar$ in the lowest order of its coupling constant expansion - such as quantities that contain only the combination $g^{2} T$ in thermal non-Abelian gauge theories - should be reliably calculable in the classical limit. Of course, there will exist quantum corrections which are suppressed by powers of the coupling constant $\alpha=g^{2} \hbar$, i.e. such a quantity will have a perturbative expansion of the form

$$
\left(g^{2} T\right)^{n}\left(c_{0}+c_{1} \alpha+c_{2} \alpha^{2}+\ldots\right)
$$

where only the coefficient $c_{0}$ is calculable classically, but the coefficients $c_{i}(i \geq 1)$ can be obtained using perturbative techniques.

Other questions also arise: What coupling constant $g$ should be used in the classical calculation? How is it renormalized? How does mass renormalization enter into the equation for the low-momentum modes that are described classically? How does the classical equation "know" that it derives its justification from the classical limit of a thermal quantum field? How does the temperature enter into the classical equation? Which mechanism ensures that the attained temperature equals that of the underlying thermal field theory, if the classically described modes thermalize due to their interaction?

Some of these questions were addressed in recent articles [12,18 20]; others remain unanswered. Here we make an attempt to address the complex of questions in its entirety, applying the method of effective field theories and the realtime formulation of finite temperature quantum field theory. Our approach leads us to the formulation of an effective dynamics for the soft modes of the quantum field in terms of a stochastic, dissipative action. The stochastic forces describe the exchange of energy and other quantum numbers with the perturbatively treated hard modes, and the dissipative terms describe the eventual approach to thermal equilibrium of the soft modes. A fluctuation-dissipation theorem ensures that the soft modes reach the same temperature as the hard modes with which they interact. We perform our analysis for the simplest nontrivial example: a massive scalar field with quartic self-interaction in $(3+1)$ dimensions. This allows us to evade the technical difficulties associated with gauge invariance and to make contact with previous work [9, 12, 18,20 .

It is worthwhile mentioning that a similar issue arises in the Euclidean formulation of thermal non-Abelian gauge theories, because thermodynamic quantities, such as the pressure or screening masses, receive contributions at a scale $\left(g^{2} T\right)^{n}$, which are essentially classical and usually cannot be calculated by means of perturbative methods. Recently, Braaten and Nieto [21.22] have shown how low-energy effective actions in Euclidean space can be utilized to systematically resolve this problem. The analogous problem in Minkowski space is more complicated, since dimensional reduction [23] does not occur in this case because at finite temperature there is no gap in the excitation spectrum. In this case the effective long-distance field theory becomes essentially classical, because quantal loop corrections are suppressed by powers of $\hbar k_{c} / T \ll 1$ where $k_{c}$ is the ultraviolet cut-off of the effective theory. However, the dynamics retains its full $(3+1)$ dimensionality.

Our manuscript is organized as follows: In the next Section we first review the concepts of effective actions and influence functionals. We then derive the influence functional for a self-coupled scalar quantum field and obtain the classical, stochastic equations of motion for the soft field modes. In Section III we show in detail how dissipation emerges from the effective equation of motion and how the proper Markovian limit of the corresponding nonlocal terms can be obtained. Section IV briefly considers the generalization of our results to gauge theories and presents a discussion of some open issues.

\section{REAL-TIME EFFECTIVE ACTION FOR CLASSICAL SOFT MODES}

\section{A. Preliminaries}

Our goal is the derivation of an effective action for the classical dynamics in Minkowski space of the low-momentum modes of a scalar quantum field $\Phi$ near thermal equilibrium. Following Bödeker, McLerran, and Smilga [18] and 
Lombardo and Mazzitelli [20], we divide the Fourier components of the field $\Phi$ into low-momentum modes with $|\mathbf{p}| \leq k_{c}$ (henceforth called "soft" modes) and high-momentum modes with $|\mathbf{p}|>k_{c}$ (called "hard" modes from now on) by defining [3 $^{3}$

$$
\Phi(\mathbf{p}, t)=\Phi(\mathbf{p}, t) \theta\left(k_{c}-|\mathbf{p}|\right)+\Phi(\mathbf{p}, t) \theta\left(|\mathbf{p}|-k_{c}\right) \equiv \phi(\mathbf{p}, t)+\varphi(\mathbf{p}, t) .
$$

It is clear from the introduction that the cutoff scale $k_{c}$ should be much smaller than the temperature T, i.e. $k_{c} \ll T$. Still $k_{c}$ may be chosen comparable with the dynamical mass scale $m^{*} \sim g T$ for the quasi-classical regime. We assume that all hard modes $\varphi$ are, and remain, thermally occupied. I $^{2}$ We do not make this assumption for the soft modes $\phi$, but we assume that their occupation number is sufficiently large to warrant a classical treatment.

We note here that this procedure is not the same as obtaining an "effective action" (in the common usage of the expression) for the expectation values of the quantum field in the presence of classical sources. The latter entails calculating the generating functional

$$
Z(J)=\int \mathcal{D} \Phi e^{i(S[\Phi]+J \phi)} \equiv e^{i W[J]}
$$

allowing for sources $J$ of the soft modes only, and then taking the Legendre transform

$$
\Gamma(\bar{\phi})=W[J]-J \frac{\delta W}{\delta J}
$$

with $\bar{\phi}=\langle\phi\rangle_{J}=\delta W / \delta J$. The effective action $\Gamma(\bar{\phi})$ contains all quantal and thermal corrections to the field expectation values.

Our definition of an "effective action" for the soft modes is 5

$$
e^{i S_{\mathrm{eff}}^{k_{c}}[\phi]}=\int \mathcal{D} \varphi e^{i S[\Phi]} \equiv \int \mathcal{D} \varphi e^{i S[\phi, \varphi]},
$$

which excludes all quantal and thermal corrections from the soft modes themselves. The two definitions only agree in the limit $k_{c} \rightarrow 0$ :

$$
S_{\text {eff }}^{k_{c}}[\phi] \stackrel{k_{c} \rightarrow 0}{\longrightarrow} \Gamma[\langle\phi\rangle] .
$$

Which approach is preferable depends on the infrared behavior of the quantum field under consideration. When it is well behaved, so that perturbation theory — possibly after resummation of a certain class of diagrams - provides an adequate description of the low-momentum sector of the field, the standard effective action $\Gamma(\phi)$ is the best choice. $\Gamma(\phi)$ already accounts for all thermal and quantal corrections and determines, in addition to the evolution of the field expectation value, all $n$-point functions of the field. On the other hand, if the low-momentum field modes are governed by truly nonperturbative physics, such as in the case of non-Abelian gauge theories at high temperature, we have to rely on a numerical treatment of the long-distance dynamics on the basis of $S_{\mathrm{eff}}^{k_{c}}[\phi]$. The major advantage then is that all the short-distance dynamics that can be treated perturbatively is already contained in the effective action $S_{\text {eff }}^{k_{c}}$, thus the numerical evaluation can concentrate on the nonperturbative sector alone where quantal corrections among the soft modes are of minor importance while classical (thermal) effects dominate.

The derivation of an effective equation of motion for the soft modes by elimination of the fast modes corresponds to a coarse graining of the quantum field. In the spirit of the general formalism of quantum statistics, the assumption of thermal equilibrium for the eliminated modes can be interpreted as one of minimal information about the microscopic quantum state of the hard modes. The general theory of coarse graining [24] then permits several statements about the nature of the effective dynamics of the soft modes:

1. The mass parameters and the coupling constants are renormalized, and new effective interactions, which are in general nonlocal, will appear.

\footnotetext{
${ }^{3}$ In the notation of ref. [18]: $\phi=\Phi_{\mathrm{L}}, \varphi=\Phi_{\mathrm{S}}$; in that of ref. [20]: $\phi=\Phi_{<,}, \varphi=\Phi_{>}$.

${ }^{4}$ This is, potentially, a weak point because the thermalization rate of hard modes is usually not significantly larger than the one of soft modes. By using a general form of the density matrix for the hard modes, the assumption of their thermalization could, in principle, be relaxed.

${ }^{5}$ Below we will generalize the effective action to the situation appropriate for real-time evolution, which requires a doubling of the degrees of freedom: $S_{\mathrm{eff}}^{k_{c}}[\phi] \rightarrow S_{\mathrm{IF}}^{k_{c}}\left[\phi, \phi^{\prime}\right]$.
} 
2. The dynamics is dissipative and contains noise terms satisfying a fluctuation-dissipation theorem. The semiclassical real-time evolution is inherently stochastic, reflecting the coupling of the soft modes to the heat reservoir of hard thermal modes.

3. The dissipative terms are nonlocal in time, giving rise to memory kernels stretching over the history of the evolution. This occurs because the heat bath needs time to respond. A change of the system (here the soft modes) influences the heat bath which in turn responds causally back onto the system.

The most convenient techniques for the formulation of the problem and the derivation of the effective action are the closed-time-path (CTP) method of Schwinger and Keldysh [25] and the influence functional (IF) method of Feynman and Vernon [26]. As discussed, e.g., in ref. [11,27] these two approaches yield identical classical equations for the expectation values of the coarse-grained field components $\phi$. In appendix A we summarize the basic idea of the influence action and its properties for a quantum mechanical system $X$ coupled to a heat bath $Q$. The influence action $S_{\mathrm{IF}}\left[\phi, \phi^{\prime} ; t_{f}\right]$ is defined as (see appendixes $\mathrm{A}$ and $\mathrm{B}$ )

$$
e^{i S_{\mathrm{IF}}\left[\phi, \phi^{\prime} ; t_{f}\right]}=\int d \varphi_{f} d \varphi_{i} d \varphi_{i}^{\prime} \int_{\varphi_{i}}^{\varphi_{f}} \mathcal{D} \varphi \int_{\varphi_{i}^{\prime}}^{\varphi_{f}} \mathcal{D} \varphi^{\prime} e^{i\left(S[\varphi]+S_{\mathrm{int}}[\phi, \varphi]-S\left[\varphi^{\prime}\right]-S_{\mathrm{int}}\left[\phi^{\prime}, \varphi^{\prime}\right]\right)} \rho_{\mathrm{h}}\left(\varphi_{i}, \varphi_{i}^{\prime} ; t_{i}\right)
$$

where $\rho_{h}\left(\varphi_{i}, \varphi_{i}^{\prime}\right)$ is the density matrix of initial configurations of the hard field modes and

$$
S[\Phi]=S[\phi]+S[\varphi]+S_{\mathrm{int}}[\phi, \varphi]
$$

is the action for the scalar field. The influence action vanishes for $\phi=\phi^{\prime}$; its variation with respect to $\phi_{\Delta}=\left(\phi-\phi^{\prime}\right)$ yields the semiclassical corrections to the equation of motion for the soft field modes $\phi$ due to their interaction with the hard modes $\varphi$.

The generating functional in the closed-time-path method is defined by introducing sources for the soft field modes only:

$$
e^{i W_{\mathrm{CTP}}\left[J, J^{\prime}\right]}=\int d \Phi_{f} d \Phi_{i} d \Phi_{i}^{\prime} \int_{\Phi_{i}}^{\Phi_{f}} \mathcal{D} \Phi \int_{\Phi_{i}^{\prime}}^{\Phi_{f}} \mathcal{D} \Phi^{\prime} e^{i\left(S[\Phi]-S\left[\Phi^{\prime}\right]+J \phi-J^{\prime} \phi^{\prime}\right)} \rho\left(\Phi_{i}, \Phi_{i}^{\prime} ; t_{i}\right),
$$

where here the limit $t_{i} \rightarrow-\infty, t_{f} \rightarrow+\infty$ is understood. Assuming that the initial density matrix of the full field factorizes into one for the hard and one for the soft modes,

$$
\rho\left(\Phi_{i}, \Phi_{i}^{\prime} ; t_{i}\right)=\rho_{\mathrm{h}}\left(\varphi_{i}, \varphi_{i}^{\prime} ; t_{i}\right) \otimes \rho_{\mathrm{s}}\left(\phi_{i}, \phi_{i}^{\prime} ; t_{i}\right)
$$

the generating functional can be expressed in terms of the influence functional as

$$
e^{i W_{\mathrm{CTP}}\left[J, J^{\prime}\right]}=\int d \phi_{f} d \phi_{i} d \phi_{i}^{\prime} \int_{\phi_{i}}^{\phi_{f}} \mathcal{D} \phi \int_{\phi_{i}^{\prime}}^{\phi_{f}} \mathcal{D} \phi^{\prime} e^{i\left(S[\phi]-S\left[\phi^{\prime}\right]+J \phi-J^{\prime} \phi^{\prime}+S_{\mathrm{IF}}\left[\phi, \phi^{\prime} ; \infty\right]\right)} \rho_{\mathrm{s}}\left(\phi_{i}, \phi_{i}^{\prime} ; t_{i}\right) .
$$

If the classical approximation can be made here, i.e. if loops involving soft momenta can be neglected and the density matrix $\rho_{\mathrm{s}}\left(\phi_{i}, \phi_{i}^{\prime} ; t_{i}\right)$ can be assumed as diagonal, the expectation values of the soft field modes are described by the effective closed-time path action

$$
\Gamma_{C T P}\left[\phi, \phi^{\prime}\right] \approx S[\phi]-S\left[\phi^{\prime}\right]+S_{\mathrm{IF}}\left[\phi, \phi^{\prime} ; \infty\right]
$$

where $\phi=\partial W_{\mathrm{CTP}} / \partial J$ and $\phi^{\prime}=\partial W_{\mathrm{CTP}} / \partial J^{\prime}$.

The main step to be taken in the derivation of effective classical equations of motion for the soft field modes are therefore: (1) calculating the influence action due to the hard modes, and (2) showing that loop contributions involving soft modes can be neglected. It is our objective in the next section to derive the influence action for the long-wavelength modes and then to discuss in the following sections the meaning of the additional terms contributing to the quasi-classical equation of motion for the soft modes in more detail.

\section{B. Influence Action}

In most cases of interest the influence action must be obtained from a suitably truncated perturbation expansion. This raises the question how far one has to go in the expansion to obtain all important physical contributions. Here we will concentrate on the case of a self-interacting scalar field with action 


$$
S[\Phi]=\int_{t_{i}}^{t} d^{4} x\left[\frac{1}{2} \partial_{\mu} \Phi \partial^{\mu} \Phi-\frac{1}{2} m^{2} \Phi^{2}-\frac{1}{4 !} g^{2} \Phi^{4}\right],
$$

where $t>t_{i}$ describes the time of observation. Details of the derivation of $S_{\mathrm{IF}}\left[\phi, \phi^{\prime}\right]$ are given in appendix B. It is our purpose here to outline the strategy and the results obtained. As will become clear, it turns out that one must include all diagrams up to order $g^{4}$ in the perturbative expansion, even if $g^{2} \ll 1$. The interaction that generates $S_{\mathrm{IF}}\left[\phi, \phi^{\prime}\right]$ is

$$
S_{\mathrm{int}}[\phi, \varphi]=-\int_{t_{i}}^{t} d^{4} x\left[\frac{1}{6} g^{2}\left(\phi^{3} \varphi+\frac{3}{2} \phi^{2} \varphi^{2}+\phi \varphi^{3}\right)+\frac{1}{4 !} g^{2} \varphi^{4}\right] .
$$

All non-vanishing diagrams contributing to $S_{\mathrm{IF}}$ up to second order in $S_{\text {int }}$ are shown in Figure 1. The diagrams (a)-(d) are the familiar one-particle irreducible diagrams appearing in the expansion of the effective action $\Gamma(\bar{\phi})$. The fifth diagram (e) vanishes in the exact limit $k_{c} \rightarrow 0$. It contributes to the coarse-grained effective action whenever $k_{c} \neq 0$. Other diagrams not listed vanish due to the constraint of (3-dimensional) momentum conservation. The inclusion of the diagrams of order $g^{4}$ is crucial, because this is the lowest order at which the real-time effective action develops an imaginary part which gives rise to stochastic terms as well as the corresponding real part will give rise to dissipative terms in the equations of motion for the soft modes. We emphasize that this property is not connected with the number of loops in the diagram: the two-loop diagram (c) as well as the one-loop diagram (d) and the tree diagram (e) contribute to noise and dissipation.

We define the real-time propagators for the hard modes as

$$
\begin{aligned}
& G_{++}^{k_{c}}(x-y)=i\langle\mathrm{~T}(\varphi(x) \varphi(y))\rangle \equiv G_{F}^{k_{c}}(x-y) \\
& G_{+-}^{k_{c}}(x-y)=i\left\langle\varphi(y) \varphi^{\prime}(x)\right\rangle \equiv G_{<}^{k_{c}}(x-y) \\
& G_{-+}^{k_{c}}(x-y)=i\left\langle\varphi^{\prime}(x) \varphi(y)\right\rangle \equiv G_{>}^{k_{c}}(x-y) \\
& G_{--}^{k_{c}}(x-y)=i\left\langle\overline{\mathrm{T}}\left(\varphi^{\prime}(x) \varphi^{\prime}(y)\right)\right\rangle,
\end{aligned}
$$

where the superscript " $k_{c}$ " indicates that these propagators are defined in the restricted space of momenta $|\mathbf{p}|>k_{c}$. As discussed in the introduction, we assume that the hard modes are thermally populated, with a simple dispersion relation

$$
\omega_{\mathbf{p}}=\sqrt{\mathbf{p}^{2}+m^{2}} .
$$

(At high temperature where the bare mass $m$ can be neglected, i.e. $m \ll g T$, choosing $k_{c}$ on the order of the dynamical mass scale $g T$ or even smaller, one can modify this dispersion by taking into account, to lowest order, the dynamically generated mass (of order $\sim g T$ ) for the hard modes themselves.)

The complex influence action $S_{\mathrm{IF}}\left[\phi, \phi^{\prime}\right]$ can be expanded in powers of the fields $\phi, \phi^{\prime}$. Up to order $g^{4}$, this expansion has the following structure (see appendix B):

$$
\begin{aligned}
& \operatorname{Re} S_{\mathrm{IF}}=-\int_{t_{i}}^{t} d^{4} x d^{4} y \sum_{N=1}^{3} \frac{1}{(2 N) !} R_{N}^{-}(x) \theta\left(x_{0}-y_{0}\right) \operatorname{Re}\left[\Gamma_{2 N}^{k_{c}}(x-y)\right] R_{N}^{+}(y) \\
& \operatorname{Im} S_{\mathrm{IF}}=-\int_{t_{i}}^{t} d^{4} x d^{4} y \sum_{N=1}^{3} \frac{1}{(2 N) !} R_{N}^{-}(x) \operatorname{Im}\left[\Gamma_{2 N}^{k_{c}}(x-y)\right] R_{N}^{-}(y)
\end{aligned}
$$

where $\Gamma_{N}^{k_{c}}$ is the amputated $2 N$-point vertex function, restricted to contributions solely from the hard modes with $|\mathbf{p}|>k_{c}$. Here we have used the abbreviations

$$
R_{N}^{ \pm}(x)=\phi(x)^{N} \pm \phi^{\prime}(x)^{N} .
$$

Up to order $g^{4}$, the vertex functions $\Gamma_{2}^{k_{c}}, \Gamma_{4}^{k_{c}}, \Gamma_{6}^{k_{c}}$ are given by the diagrams in Figure 1. All higher vertex functions vanish. $\Gamma_{2}^{k_{c}}$ is the one-particle irreducible self energy of the soft field modes as generated by the hard modes, $\Gamma_{4}^{k_{c}}$ is the one-loop correction to the bare four-particle vertex, and $\Gamma_{6}^{k_{c}}$ is a one-particle reducible six-particle vertex which vanishes in the limit $k_{c} \rightarrow 0$. Detailed expressions for the vertex functions are given in Appendix B. 


\section{Interpretation of the imaginary part of the Influence Action}

Before one can derive classical equations of motion for the soft field modes, one has to deal with the imaginary part of the influence action. The general idea of its physical significance is outlined in appendix A. It has to be interpreted as a stochastic "external" force driving the classical degrees of freedom, i.e. the soft modes. This stochastic force is an important ingredient for the equilibration of the classical modes. The standard procedure [26] is to introduce a stochastic variable $\xi$ for each imaginary contribution to $S_{\mathrm{IF}}$, making use of the identity

$$
e^{-\frac{1}{2} \chi^{T} A \chi}=[\operatorname{det}(2 \pi A)]^{-1 / 2} \int \mathcal{D} \xi e^{-\frac{1}{2} \xi^{T} A^{-1} \xi+i \chi^{T} \xi}
$$

Here $A$ is a symmetric matrix, $\chi$ and $\xi$ are vectors, and we have employed obvious vector notation. Applying this technique here we need to introduce three separate stochastic functions $\xi_{N}(x), N=1,2,3$, with the stochastic weights ${ }^{6}$

$$
P\left[\xi_{N}\right]=C_{N} \exp \left\{\int d^{4} x d^{4} y \xi_{N}(x)\left[-\frac{(2 N) !}{4 N^{2}}\left(\operatorname{Im}\left[\Gamma_{2 N}^{k_{c}}\right]\right)_{x, y}^{-1}\right] \xi_{N}(y)\right\}
$$

where $C_{N}$ are appropriate normalization constants. The influence functional then takes the form

$$
e^{i S_{\mathrm{IF}}\left[\phi, \phi^{\prime}\right]}=\int \mathcal{D} \xi_{1} \mathcal{D} \xi_{2} \mathcal{D} \xi_{3} P\left[\xi_{1}\right] P\left[\xi_{2}\right] P\left[\xi_{3}\right] e^{i \tilde{S}_{\mathrm{IF}}\left[\phi, \phi^{\prime} ; \xi_{i}\right]}
$$

with the new stochastic influence action

$$
\tilde{S}_{\mathrm{IF}}\left[\phi, \phi^{\prime} ; \xi_{1}, \xi_{2}, \xi_{3}\right]=\operatorname{Re}\left(S_{\mathrm{IF}}\left[\phi, \phi^{\prime}\right]\right)+\sum_{N=1}^{3} \frac{1}{N} \int_{t_{i}}^{t} d^{4} x R_{N}^{-}(x) \xi_{N}(x) .
$$

This expression forms the basis for the classical equations of motion for the soft modes $\phi(x)$.

\section{Classical Equations of Motion}

As we argued in the introduction, the dynamics of the soft modes under the stochastic action

$$
S[\phi]-S\left[\phi^{\prime}\right]+\tilde{S}_{\mathrm{IF}}\left[\phi, \phi^{\prime} ; \xi\right]
$$

is governed by (quasi-)classical physics, if we assume that the soft modes are sufficiently populated. As we will argue later this assumption will be justified by the full dynamics which we will derive and discuss in the following. In addition, as sketched briefly in appendix A, we note that possible initial quantum mechanical correlations in the system will decohere due to the stochastic forces acting randomly on the soft modes and so destroying those correlations (or phases) with time. We will not address this issue here, as in the present case we are convinced that the soft modes behave already classically, and thus refer the reader to the extensive literature on decoherence [10,20,29] 32]. Loop contributions to the effective action $\Gamma_{\mathrm{CTP}}[\bar{\phi}]$ from the soft modes (under the influence of $\tilde{S}_{\mathrm{IF}}$ ) are not necessarily negligible, but at high temperature they are dominated by thermal fluctuations, not quantal ones, which are relatively suppressed by a factor $\hbar k_{c} / T$. Thermal loops correspond to tree diagrams with thermally populated external legs. They can, therefore, be accounted for by averaging the classical equations of motion at the tree level over a thermal ensemble of initial states.

The classical equations of motion for the soft modes are obtained by a generalized stationary phase approximation (see appendix A) for the effective action defined on the time contour path $\mathcal{C}$ by first varying (27) with respect to $\phi_{\Delta} \equiv\left(\phi-\phi^{\prime}\right)$ and then setting $\phi=\phi^{\prime}$.

Making use of the relations

\footnotetext{
${ }^{6}$ We have chosen the index of the stochastic field to match the power of the field operators in the stochastic action. In the notation of ref. [12]: $\xi_{2} \rightarrow \xi_{1}, \xi_{1} \rightarrow \xi_{2}$; in that of ref. 20]. $\xi_{1} \rightarrow-\eta, \xi_{2} \rightarrow-\xi, \xi_{3} \rightarrow-\nu$.
} 


$$
\begin{aligned}
& \left.\frac{\delta R_{N}^{-}(x)}{\delta \phi_{\Delta}(y)}\right|_{\phi=\phi^{\prime}}=N \phi(x)^{N-1} \delta(x-y), \\
& \left.\frac{\delta R_{N}^{+}(x)}{\delta \phi_{\Delta}(y)}\right|_{\phi=\phi^{\prime}}=0,\left.\quad R_{N}^{+}(x)\right|_{\phi=\phi^{\prime}}=2 \phi(x)^{N},
\end{aligned}
$$

we find:

$$
\begin{aligned}
\varpi \phi(x) & +\tilde{m}^{2} \phi(x)+\frac{\tilde{g}^{2}}{6} \phi^{3}(x)+\sum_{N=1}^{3} \frac{1}{(2 N-1) !} \phi(x)^{N-1} \int_{t_{i}}^{t} d^{4} y \operatorname{Re}\left[\tilde{\Gamma}_{2 N}^{k_{c}}(x-y)\right] \phi(y)^{N} \\
& =\sum_{N=1}^{3} \phi(x)^{N-1} \xi_{N}(x) .
\end{aligned}
$$

We first note that the resulting equation of motion for the soft fields is explicitly causal as the real part of the influence action (21) contains a step function assuring causality, i.e.

$$
\theta\left(x_{0}-y_{0}\right) \operatorname{Re}\left[\Gamma_{2 N}^{k_{c}}(x-y)\right]
$$

defines just the response function (to lowest non-vanishing order) of the hard modes on the soft modes and thus corresponds to the familiar Kubo relation of systems near equilibrium generalized to the present case.

Secondly, the real part of the influence action is divergent and must be renormalized. Since the ultraviolet divergences of the vertex function $\Gamma_{2 N}^{k_{c}}$ are independent of the low-momentum cut-off $k_{c}$ and of the temperature, they can be absorbed in the standard counter terms for mass and coupling constant renormalization. We can, therefore, replace the divergent $\Gamma_{2 N}^{k_{c}}(x-y)$ by the regularized expression

$$
\tilde{\Gamma}_{2 N}^{k_{c}}(x-y)=\Gamma_{2 N}^{k_{c}}(x-y)-G_{2 N} \delta(x-y)
$$

where $G_{2 N}$ subtracts the temperature independent short-distance singularity of $\Gamma_{2 N}^{k_{c}=0}$. In our specific case here, $\Gamma_{2}^{k_{c}}$ and $\Gamma_{4}^{k_{c}}$ require renormalization. $\Gamma_{6}^{k_{c}}$ does not because it is finite and even vanishes in the limit $k_{c} \rightarrow 0$. Hence, $\tilde{m}$ and $\tilde{g}$ denote the renormalized mass and coupling constant, respectively, and $\tilde{\Gamma}_{2 N}^{k_{c}}$ are the renormalized vertex functions. The tadpole contributions of Figure 1(a) and 1(b) to $\tilde{\Gamma}_{2}$ contribute thermal terms to the effective mass, changing $\tilde{m}$ to $m^{*}$. The thermal effective mass $m^{*}$ will also depend on the momentum cutoff $k_{c}$ as only the mean field contributions of the hard modes are accounted for. Equation (29) is graphically represented in Figure 2. The terms on the right-hand side represent stochastic source, mass, etc., terms for the soft field modes. In particular, the term $\xi_{1}(x)$ indicates that the soft modes do not obey a source-free equation, rather, they are in constant contact with randomly fluctuating sources generated by the hard modes that have been integrated out.

The equation of motion (29) resembles a Langevin equation analogous to the situation of quantum Brownian motion. However, as it stands, it is clearly nonlocal in time, and one has to identify the appropriate Markovian limit (or approximation) of this equation. Indeed, the question arises whether a Markovian limit exists at all. The answer to this question will have a bearing on the detailed understanding of dissipation in quantum field theories. Clearly, if a Markovian limit exists, it will provide a much simpler equation which can be applied for practical purposes.

\section{DISSIPATION}

\section{A. Origin of Dissipation}

In this section we elucidate the dissipative character of the resulting contributions to the semiclassical equation of motion (29) deriving from the real parts of the influence action at order $\tilde{g}^{4}$. Only the contributions of $\operatorname{Re}\left(S_{\mathrm{IF}}^{(c)}\right)$, $\operatorname{Re}\left(S_{\mathrm{IF}}^{(d)}\right)$ and $\operatorname{Re}\left(S_{\mathrm{IF}}^{(e)}\right)$ will be responsible for dissipation, whereas the other contributions only renormalize the effective mass parameter up to order $\tilde{g}^{4}$. With this in mind we concentrate on their influence on the equation of motion (29) of the soft modes $\phi(x)$ : 


$$
\begin{aligned}
\varpi \phi(x) & +m^{* 2} \phi(x)+\frac{\tilde{g}^{2}}{6} \phi^{3}(x)+\left(\left.\sum_{i=c, d, e}(-1) \frac{\delta \operatorname{Re}\left(S_{\mathrm{IF}}^{(i)}\left[\phi, \phi_{\Delta}\right]\right)}{\delta \phi_{\Delta}(x)}\right|_{\phi_{\Delta}=0}\right) \\
& =\left(\xi_{1}(x)+\phi(x) \xi_{2}(x)+\phi(x)^{2} \xi_{3}(x)\right) .
\end{aligned}
$$

According to the general fluctuation-dissipation theorem, the three terms on the left-hand side lead to the dissipation of energy stored in the soft modes (the "system") to the hard modes (the "bath") balancing the energy gained continuously from the fluctuating force terms on the right-hand side, which were obtained from the imaginary parts of the influence functional $\operatorname{Im}\left(S_{\mathrm{IF}}^{(c)}\right), \operatorname{Im}\left(S_{\mathrm{IF}}^{(d)}\right)$, and $\operatorname{Im}\left(S_{\mathrm{IF}}^{(e)}\right)$.

From Appendix (B) we immediately find for the three dissipative terms in the sum of eq. (32):

$$
\begin{aligned}
& -\left.\frac{\delta \operatorname{Re}\left(S_{\mathrm{IF}}^{(c)}\right)}{\delta \phi_{\Delta}(x)}\right|_{\phi_{\Delta}=0}=\int_{0}^{\infty} d \tau \int d^{3} \tilde{x}\left(\frac{1}{6} \tilde{g}^{4}\right)\left(G_{>}^{k_{c}}(\tilde{\mathbf{x}}, \tau)^{3}-G_{<}^{k_{c}}(\tilde{\mathbf{x}}, \tau)^{3}\right) \phi(\mathbf{x}-\tilde{\mathbf{x}}, t-\tau), \\
& -\left.\frac{\delta \operatorname{Re}\left(S_{\mathrm{IF}}^{(d)}\right)}{\delta \phi_{\Delta}(x)}\right|_{\phi_{\Delta}=0}=\phi(x) \int_{0}^{\infty} d \tau \int d^{3} \tilde{x}\left(\frac{i}{4} \tilde{g}^{4}\right)\left(G_{>}^{k_{c}}(\tilde{\mathbf{x}}, \tau)^{2}-G_{<}^{k_{c}}(\tilde{\mathbf{x}}, \tau)^{2}\right) \phi(\mathbf{x}-\tilde{\mathbf{x}}, t-\tau)^{2}, \\
& -\left.\frac{\delta \operatorname{Re}\left(S_{\mathrm{IF}}^{(e)}\right)}{\delta \phi_{\Delta}(x)}\right|_{\phi_{\Delta}=0}=\phi(x)^{2} \int_{0}^{\infty} d \tau \int d^{3} \tilde{x}\left(-\frac{1}{12} \tilde{g}^{4}\right)\left(G_{>}^{k_{c}}(\tilde{\mathbf{x}}, \tau)-G_{<}^{k_{c}}(\tilde{\mathbf{x}}, \tau)\right) \phi(\mathbf{x}-\tilde{\mathbf{x}}, t-\tau)^{3},
\end{aligned}
$$

where we have set $x_{0}=t$.

Obviously, the contributions of the hard modes have a rather simple structure: The integration kernels are causally weighting the difference between powers of the real-time propagators $G_{>}^{k_{c}}$ and $G_{<}^{k_{c}}$. For convenience we define the memory kernels $\mathcal{M}^{(i)}$ :

$$
\begin{aligned}
& \mathcal{M}^{(c)}(\tilde{\mathbf{x}}, \tau)=\frac{1}{6} \tilde{g}^{4}\left(G_{>}^{k_{c}}(\tilde{\mathbf{x}}, \tau)^{3}-G_{<}^{k_{c}}(\tilde{\mathbf{x}}, \tau)^{3}\right), \\
& \mathcal{M}^{(d)}(\tilde{\mathbf{x}}, \tau)=\frac{i}{4} \tilde{g}^{4}\left(G_{>}^{k_{c}}(\tilde{\mathbf{x}}, \tau)^{2}-G_{<}^{k_{c}}(\tilde{\mathbf{x}}, \tau)^{2}\right), \\
& \mathcal{M}^{(e)}(\tilde{\mathbf{x}}, \tau)=-\frac{1}{12} \tilde{g}^{4}\left(G_{>}^{k_{c}}(\tilde{\mathbf{x}}, \tau)-G_{<}^{k_{c}}(\tilde{\mathbf{x}}, \tau)\right) .
\end{aligned}
$$

To further evaluate the dissipative terms (33) one has to insert an approximation for the time dependence of the soft modes $\phi(\mathbf{x}-\tilde{\mathbf{x}}, t-\tau)$ under the integrals. Previous authors [9, 12, 19, 28] have expanded $\phi$ around $t$ up to the first order gradient correction, i.e.:

$$
\phi(\mathbf{x}-\tilde{\mathbf{x}}, t-\tau)^{n} \approx \phi(\mathbf{x}, t)^{n}-n \tau \phi(\mathbf{x}, t)^{n-1} \dot{\phi}(\mathbf{x}, t) .
$$

This amounts to a quasi instantaneous approximation of the soft modes in the Heisenberg picture. If this approximation is made, the dissipative terms (33) vanish, unless an explicit width $\Gamma$ is introduced in the propagators $G_{>}^{k_{c},<\text { of }}$ the hard modes [9, 12].

We believe that this procedure obscures the true dissipative character of the memory terms. In fact, as we will see below, the Fourier frequencies of the soft modes are essential parts of the correct strategy for revealing the close connection of the expressions (33) with the more familiar on-shell scattering rates of soft modes on hard particles. Only if the soft modes oscillate periodically with some given frequency, can they scatter on the hard momentum modes or, if the frequencies allow for this, even produce hard particles. In a certain sense, the soft modes need time to scatter

\footnotetext{
${ }^{7}$ The inconsistency of this approach becomes apparent when one compares the memory time of the dissipative kernels with the characteristic oscillation period of the soft modes $\left(\left(m^{* 2}+k_{c}^{2}\right)^{-1 / 2}\right)$. From (34) the extension in past time of the kernels can roughly be estimated to be of the same order, i.e. $k_{c}^{-1}$, as it sets the the typical scale. (The memory time in a scattering process is given by the uncertainty principle as $\tau_{\text {mem }} \approx 1 /\langle\Delta E\rangle$, where $\langle\Delta E\rangle$ denotes the averaged energy transferred in the reaction. For the soft modes the energy transferred cannot be larger than $k_{c}$.) Hence the soft modes will oscillate entirely over the extension of the memory kernels.
} 
and again come on shell. The instantaneous approximation (35) clearly does not account for the oscillatory behavior of the soft modes and hence cannot properly establish the correct Markovian limit of the expressions (33). The main objective of the remainder of this section is the derivation of a well-defined Markovian form of the dissipative terms (33) that takes the oscillatory character of the soft modes in account.

The soft modes will, of course, oscillate with a certain spectrum of frequencies. This is true also for the spatial Fourier modes $\phi(\mathbf{k}, t)$. In a linear approximation one expects, however, that they oscillate with some pronounced frequency

$$
E_{k} \approx \sqrt{m^{* 2}+\mathbf{k}^{2}}
$$

This should be rigorously valid in the weak coupling regime when the soft modes propagate almost freely. Without further specifying the single-particle energy $E_{k}$, we can approximately express the soft modes at earlier times $(t-\tau)$ by their value at the time $t$ in the linear harmonic approximation

$$
\phi(\mathbf{k}, t-\tau) \approx \phi(\mathbf{k}, t) \cos E_{k} \tau-\dot{\phi}(\mathbf{k}, t) \frac{1}{E_{k}} \sin E_{k} \tau
$$

Note that the $\dot{\phi}(\mathbf{k}, t)$ contribution turns out to be essential and cannot be neglected. Comparing (37) with (35) one immediately recognizes that the harmonic approximation (37) contains much more information about the past than the instantaneous approximation (35). Equation (37) can be considered as a Markov approximation to the soft field modes in the interaction picture, where the free oscillation frequency has been extracted.

To reveal the full meaning of the three dissipative terms (33) in the equation of motion (32) of the soft modes we insert the harmonic approximation (37) and further evaluate the three terms approximately. Our strategy is completely equivalent to the quasi-particle approximation used in the more familiar kinetic theories when evaluating the collision term in transport processes [33]. A certain mode (quasi-particle) is assumed to propagate with a specific frequency, the quasi-particle energy. This approximation is valid if the spectral function of the quasi-particle is sharply peaked at this frequency. Moreover, pursuing the analogy with standard kinetic theory, the equation of motion (32) becomes instantaneous as all soft quasi-particle modes $\phi(\mathbf{k}, t)$ are evaluated at the same time. We thus obtain the Markovian limit of eq. (32) when evaluating the time integral over the memory kernels defined in (34) with the soft modes propagating according to (37).

We write (33) as

$$
\begin{aligned}
& -\left.\frac{\delta \operatorname{Re}\left(S_{\mathrm{IF}}^{(c)}\right)}{\delta \phi_{\Delta}(x)}\right|_{\phi_{\Delta}=0}=\int \frac{d^{3} k}{(2 \pi)^{3}} e^{i \mathbf{k} \cdot \mathbf{x}} \Upsilon^{(c)}(\mathbf{k}, t) \\
& -\left.\frac{\delta \operatorname{Re}\left(S_{\mathrm{IF}}^{(d)}\right)}{\delta \phi_{\Delta}(x)}\right|_{\phi_{\Delta}=0}=\phi(x) \int \frac{d^{3} k}{(2 \pi)^{3}} e^{i \mathbf{k} \cdot \mathbf{x} \Upsilon^{(d)}(\mathbf{k}, t)} \\
& -\left.\frac{\delta \operatorname{Re}\left(S_{\mathrm{IF}}^{(e)}\right)}{\delta \phi_{\Delta}(x)}\right|_{\phi_{\Delta}=0}=\phi(x)^{2} \int \frac{d^{3} k}{(2 \pi)^{3}} e^{i \mathbf{k} \cdot \mathbf{x}} \Upsilon^{(e)}(\mathbf{k}, t)
\end{aligned}
$$

with the notations

$$
\begin{aligned}
& \Upsilon^{(c)}(\mathbf{k}, t)=\theta\left(k_{c}-|\mathbf{k}|\right) \int_{0}^{\infty} d \tau \phi(\mathbf{k}, t-\tau) \mathcal{M}^{(c)}(\mathbf{k}, \tau) \\
& \Upsilon^{(d)}(\mathbf{k}, t)=\int^{k_{c}} \frac{d^{3} k_{1}}{(2 \pi)^{3}} \theta\left(k_{c}-\left|\mathbf{k}-\mathbf{k}_{1}\right|\right) \int_{0}^{\infty} d \tau \phi\left(\mathbf{k}-\mathbf{k}_{1}, t-\tau\right) \phi\left(\mathbf{k}_{1}, t-\tau\right) \mathcal{M}^{(d)}(\mathbf{k}, \tau), \\
& \Upsilon^{(e)}(\mathbf{k}, t)=\int^{k_{c}} \frac{d^{3} k_{1} d^{3} k_{2}}{(2 \pi)^{6}} \theta\left(k_{c}-\left|\mathbf{k}-\mathbf{k}_{1}-\mathbf{k}_{2}\right|\right) \int_{0}^{\infty} d \tau \phi\left(\mathbf{k}-\mathbf{k}_{1}-\mathbf{k}_{2}, t-\tau\right) \phi\left(\mathbf{k}_{1}, t-\tau\right) \phi\left(\mathbf{k}_{2}, t-\tau\right) \mathcal{M}^{(e)}(\mathbf{k}, \tau),
\end{aligned}
$$

where the momentum integrations for all soft modes are restricted by the cut-off momentum $\mathbf{k}_{c}$. We can now insert the approximate form (37) for the soft modes. It is convenient to first perform a Fourier transformation of the memory kernels

$$
\mathcal{M}^{(i)}(\mathbf{k}, \omega)=\int_{-\infty}^{\infty} d t e^{i \omega \tau} \mathcal{M}^{(i)}(\mathbf{k}, \tau)
$$


and insert the expressions for the hard propagators of Appendix B. One finds

$$
\begin{aligned}
\mathcal{M}^{(c)}(\mathbf{k}, \omega)=-\frac{\pi}{24} \tilde{g}^{4}(i) & \int_{k_{c}} \frac{d^{3} q_{1} d^{3} q_{2}}{(2 \pi)^{6}} \frac{1}{\omega_{1}} \frac{1}{\omega_{2}} \frac{1}{\omega_{3}} \theta\left(\left|\mathbf{k}-\mathbf{q}_{1}-\mathbf{q}_{2}\right|-k_{c}\right) \\
& \times\left\{\left[\left(1+n_{1}\right)\left(1+n_{2}\right)\left(1+n_{3}\right)-n_{1} n_{2} n_{3}\right] \delta\left(\omega-\omega_{1}-\omega_{2}-\omega_{3}\right)\right. \\
& +\left[\left(1+n_{1}\right) n_{2}\left(1+n_{3}\right)-n_{1}\left(1+n_{2}\right) n_{3}\right] \delta\left(\omega-\omega_{1}+\omega_{2}-\omega_{3}\right) \\
& +\left[n_{1}\left(1+n_{2}\right)\left(1+n_{3}\right)-\left(1+n_{1}\right) n_{2} n_{3}\right] \delta\left(\omega+\omega_{1}-\omega_{2}-\omega_{3}\right) \\
& +\left[n_{1} n_{2}\left(1+n_{3}\right)-\left(1+n_{1}\right)\left(1+n_{2}\right) n_{3}\right] \delta\left(\omega+\omega_{1}+\omega_{2}-\omega_{3}\right) \\
& +\left[\left(1+n_{1}\right)\left(1+n_{2}\right) n_{3}-n_{1} n_{2}\left(1+n_{3}\right)\right] \delta\left(\omega-\omega_{1}-\omega_{2}+\omega_{3}\right) \\
& +\left[\left(1+n_{1}\right) n_{2} n_{3}-n_{1}\left(1+n_{2}\right)\left(1+n_{3}\right)\right] \delta\left(\omega-\omega_{1}+\omega_{2}+\omega_{3}\right) \\
& +\left[n_{1}\left(1+n_{2}\right) n_{3}-\left(1+n_{1}\right) n_{2}\left(1+n_{3}\right)\right] \delta\left(\omega+\omega_{1}-\omega_{2}+\omega_{3}\right) \\
& \left.+\left[n_{1} n_{2} n_{3}-\left(1+n_{1}\right)\left(1+n_{2}\right)\left(1+n_{3}\right)\right] \delta\left(\omega+\omega_{1}+\omega_{2}+\omega_{3}\right)\right\}
\end{aligned}
$$

where $\omega_{1}=\omega_{\mathbf{q}_{1}}, \omega_{2}=\omega_{\mathbf{q}_{2}}, \omega_{3}=\omega_{\mathbf{k}-\mathbf{q}_{1}-\mathbf{q}_{2}}$ and $n_{\alpha}=n\left(\omega_{\mathbf{q}_{\alpha}}\right)$, and

$$
\begin{aligned}
\mathcal{M}^{(d)}(\mathbf{k}, \omega)=-\frac{\pi}{8} \tilde{g}^{4}(i) & \int_{k_{c}} \frac{d^{3} q}{(2 \pi)^{3}} \frac{1}{\omega_{1}} \frac{1}{\omega_{2}} \theta\left(|\mathbf{k}-\mathbf{q}|-k_{c}\right) \\
\times & \left\{\left[\left(1+n_{1}\right)\left(1+n_{2}\right)-n_{1} n_{2}\right] \delta\left(\omega-\omega_{1}-\omega_{2}\right)\right. \\
& +\left[n_{1}\left(1+n_{2}\right)-\left(1+n_{1}\right) n_{2}\right] \delta\left(\omega+\omega_{1}-\omega_{2}\right) \\
& +\left[\left(1+n_{1}\right) n_{2}-n_{1}\left(1+n_{2}\right)\right] \delta\left(\omega-\omega_{1}+\omega_{2}\right) \\
& \left.+\left[n_{1} n_{2}-\left(1+n_{1}\right)\left(1+n_{2}\right)\right] \delta\left(\omega+\omega_{1}+\omega_{2}\right)\right\}
\end{aligned}
$$

where $\omega_{1}=\omega_{\mathbf{q}}, \omega_{2}=\omega_{\mathbf{k}-\mathbf{q}}$, and

$$
\begin{aligned}
\mathcal{M}^{(e)}(\mathbf{k}, \omega)= & -\frac{\pi}{12} \tilde{g}^{4}(i) \frac{1}{\omega_{\mathbf{k}}} \theta\left(|\mathbf{k}|-k_{c}\right) . \\
& \left\{\left[\left(1+n\left(\omega_{\mathbf{k}}\right)\right)-n\left(\omega_{\mathbf{k}}\right)\right] \delta\left(\omega-\omega_{\mathbf{k}}\right)+\left[n\left(\omega_{\mathbf{k}}\right)-\left(1+n\left(\omega_{\mathbf{k}}\right)\right)\right] \delta\left(\omega+\omega_{\mathbf{k}}\right)\right\} .
\end{aligned}
$$

The memory kernels $\mathcal{M}^{(i)}(\mathbf{k}, \omega)$ have a very simple structure. They all are purely imaginary and satisfy the relation

$$
\mathcal{M}^{(i)}(\mathbf{k}, \omega)=-\mathcal{M}^{(i)}(\mathbf{k},-\omega) .
$$

Moreover, the kernels are directly related to the discontinuities of the respective self-energy insertions of the hard modes. For a detailed discussion and interpretation of such discontinuities in finite temperature field theory we refer the reader to the article by Weldon [34]. From the expressions (41-43) we see that each kernel is composed of two contributions: the direct part $\Gamma_{d}(\mathbf{k}, \omega)$, or the "loss" term, and the inverse part $\Gamma_{i}(\mathbf{k}, \omega)$, or "gain" term. Following [34], we write

$$
i \mathcal{M}(\mathbf{k}, \omega) \propto \Gamma_{d}(\mathbf{k}, \omega)-\Gamma_{i}(\mathbf{k}, \omega),
$$

where $\Gamma_{d}$ and $\Gamma_{i}$, respectively, are positive and can be identified with the decay rate of a quasi-particle with momentum $\mathbf{k}$ and energy $\omega$ into the allowed open channels or the production rate due to the inverse processes. In addition, the detailed balance relation

$$
\frac{\Gamma_{d}(\omega)}{\Gamma_{i}(\omega)}=\exp (\omega / T)
$$

follows immediately if the distribution function $n(\omega)$ of the hard modes is assumed to be in thermal equilibrium.

This identification represents an important step toward understanding the dissipative nature of the terms (33). Intuitively, we expect dissipation to be closely related to the on-shell scattering rates of the soft modes when interacting with the hard thermal particles. We have to manipulate the terms further in order to fully expose this connection.

We now insert the approximation (37) for the soft quasi-particle modes into the semi-infinite integrals $\Upsilon^{(i)}(\mathbf{k}, t)$ given by (39). In order to evaluate the time integral we express the memory kernels by their Fourier transform $\mathcal{M}^{(i)}(\mathbf{k}, \omega)$ and make use of the relation

$$
\int_{0}^{\infty} d \tau e^{i(\gamma-\omega) \tau}=i \lim _{\epsilon \rightarrow 0^{+}} \frac{1}{(\gamma-\omega)+i \epsilon}=i P \frac{1}{\gamma-\omega}+\pi \delta(\gamma-\omega) .
$$


The remainder of the calculation is straightforward but cumbersome. One has to express the sine and cosine contributions by exponentials and collect all terms. Relation (44) helps in identifying certain pairwise identical contributions. The final result reads

$$
\begin{aligned}
& \Upsilon^{(c)}(\mathbf{k}, t)=\phi(\mathbf{k}, t) \theta\left(k_{c}-|\mathbf{k}|\right) \int \frac{d \omega}{2 \pi} i \mathcal{M}^{(c)}(\mathbf{k}, \omega) P \frac{1}{E_{k}-\omega}+\dot{\phi}(\mathbf{k}, t) \theta\left(k_{c}-|\mathbf{k}|\right) \frac{1}{2 E_{k}} i \mathcal{M}^{(c)}\left(\mathbf{k}, E_{k}\right) \\
& \Upsilon^{(d)}(\mathbf{k}, t)=\int^{k_{c}} \frac{d^{3} k_{1}}{(2 \pi)^{3}} \theta\left(k_{c}-\left|\mathbf{k}-\mathbf{k}_{1}\right|\right)\left\{\phi\left(\mathbf{k}-\mathbf{k}_{1}, t\right) \phi\left(\mathbf{k}_{1}, t\right) \frac{1}{2} \int \frac{d \omega}{2 \pi} i \mathcal{M}^{(d)}(\mathbf{k}, \omega)\right. \\
& \times\left(P \frac{1}{E_{\mathbf{k}-\mathbf{k}_{1}}+E_{k_{1}}-\omega}+P \frac{1}{E_{\mathbf{k}-\mathbf{k}_{1}}-E_{k_{1}}-\omega}\right) \\
& +\dot{\phi}\left(\mathbf{k}-\mathbf{k}_{1}, t\right) \dot{\phi}\left(\mathbf{k}_{1}, t\right) \frac{1}{2 E_{\mathbf{k}-\mathbf{k}_{1}} E_{k_{1}}} \int \frac{d \omega}{2 \pi} i \mathcal{M}^{(d)}(\mathbf{k}, \omega) \\
& \left.\times\left(-P \frac{1}{E_{\mathbf{k}-\mathbf{k}_{1}}+E_{k_{1}}-\omega}+P \frac{1}{E_{\mathbf{k}-\mathbf{k}_{1}}-E_{k_{1}}-\omega}\right)\right\} \\
& +\int^{k_{c}} \frac{d^{3} k_{1}}{(2 \pi)^{3}} \dot{\phi}\left(\mathbf{k}-\mathbf{k}_{1}, t\right) \phi\left(\mathbf{k}_{1}, t\right) \frac{1}{4 E_{\mathbf{k}-\mathbf{k}_{1}}} \theta\left(k_{c}-\left|\mathbf{k}-\mathbf{k}_{1}\right|\right) \\
& \times\left(i \mathcal{M}^{(d)}\left(\mathbf{k}, E_{\mathbf{k}-\mathbf{k}_{1}}+E_{k_{1}}\right)+i \mathcal{M}^{(d)}\left(\mathbf{k}, E_{\mathbf{k}-\mathbf{k}_{1}}-E_{k_{1}}\right)\right), \\
& \Upsilon^{(e)}(\mathbf{k}, t)=\int^{k_{c}} \frac{d^{3} k_{1} d^{3} k_{2}}{(2 \pi)^{6}} \theta\left(k_{c}-\left|\mathbf{k}_{3}\right|\right)\left\{\phi\left(\mathbf{k}_{1}, t\right) \phi\left(\mathbf{k}_{2}, t\right) \phi\left(\mathbf{k}_{3}, t\right) \frac{1}{4} \int \frac{d \omega}{2 \pi}(i) \mathcal{M}^{(e)}(\mathbf{k}, \omega)\right. \\
& \times\left(P \frac{1}{E_{1}+E_{2}+E_{3}-\omega}+P \frac{1}{E_{1}+E_{2}-E_{3}-\omega}+2 P \frac{1}{E_{1}-E_{2}+E_{3}-\omega}\right) \\
& +\quad \dot{\phi}\left(\mathbf{k}_{1}, t\right) \dot{\phi}\left(\mathbf{k}_{2}, t\right) \phi\left(\mathbf{k}_{3}, t\right) \frac{3}{4 E_{1} E_{2}} \int \frac{d \omega}{2 \pi} i \mathcal{M}^{(e)}(\mathbf{k}, \omega) \\
& \left.\times\left(-P \frac{1}{E_{1}+E_{2}+E_{3}-\omega}-P \frac{1}{E_{1}+E_{2}-E_{3}-\omega}+2 P \frac{1}{E_{1}-E_{2}+E_{3}-\omega}\right)\right\} \\
& +\int^{k_{c}} \frac{d^{3} k_{1} d^{3} k_{2}}{(2 \pi)^{6}} \theta\left(k_{c}-\left|\mathbf{k}_{3}\right|\right)\left\{\phi\left(\mathbf{k}_{1}, t\right) \phi\left(\mathbf{k}_{2}, t\right) \dot{\phi}\left(\mathbf{k}_{3}, t\right) \frac{3}{8 E_{3}}\right. \\
& \times\left(i \mathcal{M}^{(e)}\left(\mathbf{k}, E_{1}+E_{2}+E_{3}\right)+i \mathcal{M}^{(e)}\left(\mathbf{k},-E_{1}-E_{2}+E_{3}\right)+2 i \mathcal{M}^{(e)}\left(\mathbf{k}, E_{1}-E_{2}+E_{3}\right)\right) \\
& +\dot{\phi}\left(\mathbf{k}_{1}, t\right) \dot{\phi}\left(\mathbf{k}_{2}, t\right) \dot{\phi}\left(\mathbf{k}_{3}, t\right) \frac{1}{8 E_{1} E_{2} E_{3}} \\
& \times\left(i \mathcal{M}^{(e)}\left(\mathbf{k},-E_{1}-E_{2}-E_{3}\right)+i \mathcal{M}^{(e)}\left(\mathbf{k}, E_{1}+E_{2}-E_{3}\right)\right. \\
& \left.\left.+2 i \mathcal{M}^{(e)}\left(\mathbf{k}, E_{1}-E_{2}+E_{3}\right)\right)\right\} \text {. }
\end{aligned}
$$

where $\mathbf{k}_{3}=\mathbf{k}-\mathbf{k}_{1}-\mathbf{k}_{2}$.

One notices that the contributions with an odd power in $\dot{\phi}$ contain the on-shell reaction rates (41, 43). Thus we expect them to describe the dissipative aspects of the equation of motion for the soft modes as energy is transferred by scattering (or production) processes between the soft and hard modes. Since this interpretation is most obvious for the first term $\Upsilon^{(c)}$, let us start by discussing this term in more detail. Fourier transforming the equation of motion (32) for the mode $\mathbf{k}$, we find from (38) that $\operatorname{Re}\left(S_{\mathrm{IF}}^{(c)}\right)$ contributes on the right hand side as

$$
\begin{aligned}
& \ddot{\phi}(\mathbf{k}, t)+\mathbf{k}^{2} \phi(\mathbf{k}, t)+\tilde{m}^{2} \phi(\mathbf{k}, t)+\frac{\tilde{g}^{2}}{6} \int^{k_{c}} \frac{d^{3} k_{1} d^{3} k_{2}}{(2 \pi)^{6}} \theta\left(k_{c}-\left|\mathbf{k}-\mathbf{k}_{1}-\mathbf{k}_{2}\right|\right) \phi\left(\mathbf{k}_{1}, t\right) \phi\left(\mathbf{k}_{2}, t\right) \phi\left(\mathbf{k}-\mathbf{k}_{1}-\mathbf{k}_{2}, t\right) \\
& \quad+\phi(\mathbf{k}, t) P \int \frac{d \omega}{2 \pi} \frac{i \mathcal{M}^{(c)}(\mathbf{k}, \omega)}{E_{k}-\omega}+\dot{\phi}(\mathbf{k}, t) \frac{i \mathcal{M}^{(c)}\left(\mathbf{k}, E_{k}\right)}{2 E_{k}}+\ldots=\xi_{1}(\mathbf{k}, t)+\ldots .
\end{aligned}
$$

The first additional term can be readily interpreted as momentum dependent contribution to the mass term (after proper renormalization). The second term has the familiar structure of a velocity dependent damping term, i.e. $\eta \dot{\phi}(\mathbf{k}, t)$. From (41) and the detailed balance relation (46) we find that the friction coefficient

$$
\eta^{(c)}(\mathbf{k})=\frac{i \mathcal{M}^{(c)}\left(\mathbf{k}, E_{k}\right)}{2 E_{k}} \geq 0
$$


is in fact positive. It is exactly related to the rate $\left(\Gamma_{d}-\Gamma_{i}\right)$ with which a small disturbance relaxes towards equilibrium by coupling to the various open channels [34]. The damping rate for the soft modes in this particular channel follows immediately as

$$
\gamma^{(c)}(\mathbf{k})=\frac{1}{2} \eta^{(c)}(\mathbf{k})
$$

as the left hand side of eq. (51) has the well known solution of a weakly damped oscillator

$$
\phi(\mathbf{k}, t) \sim e^{-\eta^{(c)}(\mathbf{k}) t / 2} \cos \left(E_{k}^{2}-\frac{1}{4} \eta^{(c)}(\mathbf{k})^{2}\right)^{1 / 2} t .
$$

Generalizing this observation we expect a similar interpretation of the two other dissipative terms, $\Upsilon^{(d)}$ and $\Upsilon^{(e)}$, to hold: (1) The principal value contributions give rise to a (generally momentum dependent) vertex renormalization and to a new (again momentum dependent) six-point vertex; (2) the on-shell contributions can be associated with damping processes involving two hard particles and two soft modes or involving one hard particle interacting with three soft modes, respectively. In Appendix $\mathrm{C}$ we present a further analysis of the contributions from $\operatorname{Re}\left(S_{\mathrm{IF}}^{(d)}\right)$ and $\operatorname{Re}\left(S_{\mathrm{IF}}^{(e)}\right)$, where a bridge is cast to the evaluation of the discontinuities by means of soft quasi-particle propagators rather than soft fields. In particular the amplitude squared is easily identified with the quasi-particle density of the soft modes, i.e.

$$
|\phi(\mathbf{k}, t)|^{2}=\phi(\mathbf{k}, t) \phi(-\mathbf{k}, t) \approx \frac{1}{E_{k}} N(\mathbf{k}, t) \approx \frac{1}{E_{k}}(1+N(\mathbf{k}, t))
$$

in the quasi-classical regime where the soft modes are strongly populated. One finds:

$$
\begin{aligned}
& \eta^{(d)}(\mathbf{k}, t) \approx \frac{1}{4 E_{k}} \int^{k_{c}} \frac{d^{3} k_{1}}{(2 \pi)^{3}}\left|\phi\left(\mathbf{k}_{1}, t\right)\right|^{2} \\
& \times\left(i \mathcal{M}^{(d)}\left(\mathbf{k}+\mathbf{k}_{1}, E_{k}+E_{k_{1}}\right)+i \mathcal{M}^{(d)}\left(\mathbf{k}+\mathbf{k}_{1}, E_{k}-E_{k_{1}}\right)\right) \\
& \eta^{(e)}(\mathbf{k}, t) \approx \frac{3}{4 E_{k}} \int^{k_{c}} \frac{d^{3} k_{1} d^{3} k_{2}}{(2 \pi)^{6}}\left|\phi\left(\mathbf{k}_{1}, t\right)\right|^{2}\left|\phi\left(\mathbf{k}_{2}, t\right)\right|^{2} \\
& \quad \times\left(i \mathcal{M}^{(e)}\left(\mathbf{k}+\mathbf{k}_{1}+\mathbf{k}_{2}, E_{k}+E_{k_{1}}+E_{k_{2}}\right)\right. \\
& \quad+i \mathcal{M}^{(e)}\left(\mathbf{k}+\mathbf{k}_{1}+\mathbf{k}_{2}, E_{k}-E_{k_{1}}-E_{k_{2}}\right) \\
&\left.+2 i \mathcal{M}^{(e)}\left(\mathbf{k}+\mathbf{k}_{1}+\mathbf{k}_{2}, E_{k}+E_{k_{1}}-E_{k_{2}}\right)\right) .
\end{aligned}
$$

These are the obvious generalizations of (52) and correspond to the friction coefficients for the possible on-shell scattering or production processes among two soft and two hard modes and three soft modes and one hard mode, respectively. We note, however, that these coefficients do not need to be positive for all times. From (45) and (46) it follows that $\mathcal{M}$ is positive for $\omega>0$, whereas $\mathcal{M}=-\mathcal{M}(|\omega|)$ becomes negative for $\omega<0$. Hence the second integrand of (55) as well as the last two of (56) can become negative, so that in principle "anti-damping" can occur in some cases. This is not a conceptual difficulty. Suppose one would prepare an initial system where a few modes are much less populated than all the others. These special modes should grow in magnitude by scattering processes either due to the direct $\phi^{3}$-term in (32), or by scattering processes including other soft modes and hard particles. Energy is then transferred from the other soft modes to those few and into the reservoir of hard particles.

Summarizing this section, we succeeded in properly identifying the origin of dissipation from on-shell scattering processes of soft quasi-particles. Dissipation corresponds directly to the imaginary parts of the self energy insertions $\left[\operatorname{Re}_{2 N}^{k_{c}}\right](\mathbf{k}, \omega)$ in the response term of eq. (29) — which should not be mixed up with the imaginary parts of (21) of the effective action - as one would have expected from standard finite temperature field theory. Furthermore instantaneous, but momentum dependent dissipation coefficients have been derived in the proper Markovian limit of the effective terms (33).

\section{B. The limit $k_{c} \rightarrow 0$}

To draw comparison with other work [9, 12,14] it is useful to consider the limit $k_{c} \rightarrow 0$ for the scalar and massive theory: 


$$
\phi(\mathbf{x}, t) \longrightarrow \phi(t) ; \quad \phi(\mathbf{k}, t) \longrightarrow \phi(t)(2 \pi)^{3} \delta^{3}(\mathbf{k}) .
$$

The analysis follows in complete analogy to the preceding section so that we skip the intermediate steps and state the final results $\left(k_{c} \rightarrow 0\right)$ :

$$
\begin{aligned}
\eta^{(c)} & \longrightarrow \frac{1}{2 E_{0}} i \mathcal{M}^{(c)}\left(0, E_{0}\right) \geq 0, \\
\eta^{(d)}(t) & \longrightarrow \phi(t)^{2} \frac{1}{4 E_{0}} i \mathcal{M}^{(d)}\left(0,2 E_{0}\right) \\
& =\phi(t)^{2} \frac{\pi \tilde{g}^{4}}{32 E_{0}} \int_{k_{c} \rightarrow 0} \frac{d^{3} q}{(2 \pi)^{3}} \frac{1}{\omega_{q}^{2}}\left(1+2 n\left(\omega_{q}\right)\right) \delta\left(E_{0}-\omega_{q}\right) \geq 0, \\
\eta^{(e)}(t) & \longrightarrow 0 .
\end{aligned}
$$

Here $E_{0}$ denotes the infrared limit of the dispersion relation $E_{\mathbf{k}}$ of the soft modes. The first dissipation coefficient due to the "sunset" graph (Figure 1c) contains contributions due to scattering as well as particle production. The second coefficient is only nonvanishing when producing hard particles, i.e. when the low momentum mode $\phi(t)$ possesses Fourier frequencies that allow for such a dissipative channel. The last term vanishes as the hard propagator is restricted to momenta $|\mathbf{q}|>k_{c}=0$.

The dynamical mass $\left(m^{*}\right)^{2}$ to order $\tilde{g}^{2}$ is given by the tadpole diagram (a) in Figure 1: $\left(m^{*}\right)^{2}=\tilde{g}^{2} T^{2} / 24$, which is the dominant contribution to the mass in the high temperature limit, i.e. when $\tilde{m} \ll m^{*}$. Inserting this for the dispersion relation of the energy, i.e. $E_{0}=m^{*}$, the damping coefficient $\eta^{(c)}$ is exactly related to twice the damping rate of plasmons in $\Phi^{4}$ theory [35] 37]:

$$
\eta^{(c)}=2 \gamma\left(m^{*}, 0\right)=\frac{\tilde{g}^{3} T}{32 \sqrt{24} \pi},
$$

as one would have expected by our arguments given at the end of the last section.

We admit that our approximate treatment of a single harmonic approximation (37) for the soft mode may fail when $\phi(t)$ contains a whole set of frequencies, e.g. if one thinks of unstable phase transitions where $\phi(t)$ acts as the relevant order parameter. In this case, however, one can work with the explicit temporally nonlocal effective terms which one can easily write down from the expressions of the memory kernels at $\mathbf{k}=0$. Such a procedure was undertaken by Boyanovsky et al. 14]. At zero temperature the authors examined in detail the damping associated with the contribution of the 'fish-graph' (d) in a more self-consistent treatment by resumming its influence on the evolution of the (ensemble) averaged order parameter $\langle\phi(t)\rangle$. They found that the dissipation was strongest in the Goldstone sector when (nearly) massless particles can be produced. Such a reasoning follows also immediately from our result (59) as then $\eta^{(d)}$ becomes nonvanishing even for $k_{c} \rightarrow 0$. As outlined above, we share the authors' point of view that an instantaneous approximation as assumed in (35) is not valid and cannot correctly account for the dissipation contained in the nonlocal expressions, such as (33).

\section{A remark on the fluctuation-dissipation relation}

The dissipative terms and the noise terms are related by fluctuation-dissipation relations. From Appendices A and $\mathrm{B}$ we see that the memory kernels $\mathcal{M} \equiv D_{R}$ (the response functions) and the noise kernels $\nu \equiv D_{I}$ are closely related (cf. their definitions (A21) or (B11, B12, B13)). On general grounds intimate relations among these kernels exist, known as the generalized fluctuation-dissipation relations (FDR). However, their explicit realizations are quite complicated. For a detailed derivation which can be readily applied to our case we refer to [10]. Because of the symmetry relations $\mathcal{M}(\mathbf{k}, \omega)=-\mathcal{M}(\mathbf{k},-\omega)$ and $\nu(\mathbf{k}, \omega)=\nu(\mathbf{k},-\omega)$ one defines a new kernel $\gamma$ as

$$
\mathcal{M}(\tau)=\frac{d}{d \tau} \gamma(\tau)
$$

Then a relation among $\gamma$ and $\nu$ can be obtained in the form

$$
\nu(\tau)=\int_{-\infty}^{\infty} d s K(\tau-s) \gamma(s)
$$

which is just the generalized FDR. In order to see how $K$ can be derived, we make use of (46) together with (62) to write 


$$
\gamma(\omega)=\frac{i}{\omega} \mathcal{M}(\omega) \propto \frac{1}{\omega}\left(\Gamma_{d}(\mathbf{k}, \omega)-\Gamma_{i}(\mathbf{k}, \omega)\right) .
$$

For the noise kernels a similar identification holds (compare the explicit forms given in Appendix B)

$$
\nu(\omega) \propto \Gamma_{d}(\mathbf{k}, \omega)+\Gamma_{i}(\mathbf{k}, \omega) .
$$

(The Fourier transform of the noise kernel is thus always non-negative.) Because of (46), one finds that

$$
K(\omega)=\frac{\gamma(\omega)}{\nu(\omega)}=\frac{1}{\omega} \frac{\exp (\hbar \omega / T)-1}{\exp (\hbar \omega / T)+1} .
$$

Now the important point to stress is that the frequencies involved for the soft modes are much smaller than the temperature T, i.e. $E_{k} \ll T$ for $k_{c} \ll T$. Hence only those low frequencies are relevant for the kernels $\mathcal{M}, \nu$ and $\gamma$, as also only those noises containing small frequencies can influence the dynamics of the soft modes. For $\nu \equiv I$ this follows in particular if one rewrites the Gaussian distribution of the noise, e.g. (A29), in the frequency domain as

$$
P[\xi(\omega)]=\frac{1}{N} \exp \left(-\frac{1}{2} \int d \omega \xi(\omega) I^{-1}(-\omega) \xi(-\omega)\right) .
$$

If one thus considers only the relevant low frequency part of the kernels, $K$ becomes effectively constant and thus local in time:

$$
K(s) \stackrel{\omega \ll T}{\longrightarrow} 2 T / \hbar,
$$

the famous Kubo identity. Such a simple relation always occurs if the system behaves classically [40]. In fact, as we have argued before, the soft modes should satisfy this condition.

The FDR ensures that the soft modes approach thermal equilibrium precisely at the temperature $T$ of the hard modes, when they evolve under the equation of motion (32). The presence of these terms is essential, as it forces the soft modes to thermalize at $T$ independent of their initial configuration. In particular, the noise terms will continuously 'heat' the system (for field theories see ref. [41]) whereas the dissipative part of the response function counteracts. Equilibrium is achieved when the system has thermalized to the temperature dictated by the bath. This aspect is not important if one only wants to study the time evolution of thermal configurations of the soft modes. However, for the study of configurations far off thermal equilibrium the presence of both noise and friction terms is crucial, as it ensures that the soft modes will become populated thermally, i.e. their amplitudes become large. This justifies our basic assumption.

This remark bears relevance to the issue whether thermal masses can suppress chaos in certain nonlinear classical field theories as suggested by Blaizot and Iancu [42]. They argued that small amplitude, homogeneous field configurations in non-Abelian gauge theories oscillate regularly due to their thermal mass, and only large amplitude waves become chaotic. Our above result shows that soft modes do not remain of small amplitude when stochastic forces are included in their description, rather their amplitude always approaches the thermal limit. It would be interesting to study this phenomenon, e.g. in the case of two massless scalar fields $\phi$ and $\chi$, which are coupled through an interaction of the form $g^{2} \phi^{2} \chi^{2}$. This theory exhibits chaos in the naive classical limit. Whether the effective stochastic classical theory for soft field modes remains chaotic in spite of thermal mass generation remains to be seen.

\section{Memory effects}

As discussed at length above, the real part of the influence action yields nonlocal, dissipative terms in the effective classical equation of motion (32). For further exploration of the nature of dissipation we made the Markov ansatz (37) to calculate the dissipation coefficients. If the strength of the soft modes, $\sqrt{|\phi(\mathbf{k}, t)|^{2}}$, also changes rapidly with time (e.g. in the strong coupling regime) the Markov limit may not be valid. This can happen as the memory kernels $\mathcal{M}(\tau)$ have support in the past roughly given by the scattering time of the individual processes. To avoid such interference effects one has to choose the appropriate cutoff $k_{c}$ large enough to restrict the temporal extent of the kernels. This becomes relevant in the case of massless theories in the strong coupling regime. However, the validity of the quasi-classical behavior of the soft modes is then no longer warranted. 


\section{E. Infrared behavior}

We restate the quasi-classical equation of motion for the soft modes $\phi(\mathbf{k}, t)$ in their approximate instantaneous form:

$$
\begin{gathered}
\frac{\partial^{2} \phi}{\partial t^{2}}+\left(\mathbf{k}^{2}+\tilde{m}^{2}+\sum_{i=a, b, c} \mu_{1, k_{c}}^{i}\right) \phi+\left(\frac{\tilde{g}^{2}}{6}+\mu_{2, k_{c}}^{(d)}\right) \otimes \phi^{3}+ \\
\mu_{3, k_{c}}^{(e)} \otimes \phi^{5}+\sum_{i=c, d, e} \eta_{k_{c}}^{(i)} \dot{\phi} \approx \sum_{N=1}^{3} \xi_{n} \otimes \phi^{N-1},
\end{gathered}
$$

where $\otimes$ denotes a convolution in momentum space. The coefficients $\mu_{i}$ generally depend on $\mathbf{k}$ and $t$, as well as on the momentum cut-off $k_{c}$. Explicit calculation shows that the leading cut-off dependence of $\mu_{1}^{k}$ derives from the thermal one-loop contribution [18] (see also [39]):

$$
\mu_{1, k_{c}}^{(a)} \longrightarrow \frac{\tilde{g}^{2} T^{2}}{24}-\frac{\tilde{g}^{2} k_{c} T}{4 \pi} \quad \text { for } \tilde{m}=0 .
$$

The vacuum contribution vanishes quadratically for small $k_{c}$. The same is true for the one-loop correction to the coupling constant [39]:

$$
\mu_{2, k_{c}}^{(d)} \stackrel{\mathbf{k} \rightarrow 0}{\approx}-\frac{3 \tilde{g}^{4} T}{16 \pi m^{*}}\left(1-\frac{2 k_{c}}{\pi m^{*}}\right) \stackrel{\tilde{m} \rightarrow 0}{\longrightarrow} \frac{3 \sqrt{6}}{8 \pi} \tilde{g}^{3}\left(1+\frac{3 k_{c}}{\pi T}\right)-\frac{9 \tilde{g}^{2} k_{c}}{\pi^{2} T}
$$

where $m^{* 2}=\tilde{m}^{2}+\mu_{1}(0)$ is the effective thermal mass. As discussed in 18, the cut-off dependence of $\mu_{1}$ is exactly balanced by the dynamically generated mass due to the self-interaction among the soft modes, if these are also in thermal equilibrium. The same holds for the other $k_{c}$-dependent constants in (69). If $\Gamma_{2 N}^{k_{c}=0}$ contains an infrared divergence, as often happens in massless quantum field theories, it is necessary to "resum" the thermal self-energy by including it into the propagator of the hard modes [35,38]. (See also 12 for an extended discussion of this procedure.)

\section{CONCLUSIONS AND OUTLOOK}

In summary, we have succeeded in deriving a consistent set of temporally local transport equations for the longdistance modes of a self-coupled scalar field. Our approach is based on the analysis of time scales showing that the soft field modes, at high temperature and weak coupling, oscillate on a time scale shorter than the characteristic damping time. A temporally local (Markovian) equation can therefore only be derived for the Fourier components of the soft field modes, i.e. the occupation amplitudes, but not for the field itself. In the spirit of quantum field theory, this corresponds to the use of the interaction picture rather than the Heisenberg picture.

As a result of our modified approach, we obtain finite values for the dissipation coefficients without the need to introduce an explicit damping for the hard field modes. The dominant damping mechanism for the infrared field mode $\left(k_{c}=0\right)$ is generally given by the two-loop "sunset" diagram, corresponding to emission or absorption of the soft mode on hard thermal quanta. This differs from the analysis of Gleiser and Ramos [12] who found that soft-mode damping is dominated by scattering on hard particles in the traditional approach of Morikawa [9].

How do our results generalize to other field theories? Most interesting quantum field theories, especially gauge theories, contain three-particle vertices rather than, or in addition to, the four-point coupling investigated here. In these cases, there exists the standard one-loop diagram, shown in Figure 3a for the $\phi^{3}$-theory. The real part associated to this diagram has an imaginary part (in the $(k, \omega)$-representation) in the space-like domain, corresponding to the fluctuations in the mean field at space-like distances induced by hard thermal particles. While these fluctuations do not directly produce damping of on-shell soft field modes, they provide a mechanism for such damping, when coupled back into the nonlinear equation for these modes. Graphically, to lowest order, this mechanism will generate a higher order diagram as shown in Figure 3b. This corresponds, similar to our arguments presented in Appendix C, to the imaginary part of the two loop diagram (also shown in Figure 3b) when cut through the hard thermal loop and the soft field line. This analysis of mean field damping in the case of three-point vertices is completely analogous to that performed by Braaten and Pisarski [51] for the damping of soft modes in non-Abelian gauge theories.

Non-Abelian gauge fields at finite temperature would present an interesting application of the methods discussed so far, because their infrared sector remains non-perturbative even at high temperature due to the absence of perturbative 
screening of magnetostatic fields. This phenomenon has been widely studied in the Euclidean formalism, and its resolution by a sequence of effective actions has been proposed [21,22. As mentioned in the Introduction, the situation is more delicate in the real-time formalism because of the lack of a gap in the excitation spectrum.

The emergence of a finite correlation length in the magnetostatic sector is essentially a classical phenomenon which occurs at the momentum scale $g^{2} T$ 43 46]. It should be fully contained in the classical effective action obtained by integrating out hard modes with momenta $|\mathbf{p}|>k_{c}>g^{2} T$. In fact, our discussion in Section II.E suggests that we should choose $k_{c} \sim g T$ in non-Abelian gauge theories because the thermal gauge boson mass $m^{*}$ is of order $g T$. This mass itself is of semiclassical origin [47 49, but requires the use of the Bose distribution for the occupation number of hard modes and hence is sensitive to quantum physics.

The problem in gauge theories is that one cannot employ a low-momentum cut-off $k_{c}$ because it is not gauge invariant. A lattice formulation provides a gauge invariant cut-off through the lattice spacing $a$, roughly corresponding to $k_{c}=\pi / a$, but it violates rotational invariance. As pointed out in ref. [18] this necessitates the introduction of rotationally non-invariant counterterms. An infrared cut-off scheme that violates neither gauge nor Lorentz invariance is therefore preferable. Such a scheme, based on the proper time representation of the one-loop effective action, has recently been proposed $[50]$.

We found it convenient to formulate the effective equations of motion (51) for the soft field modes in momentum space. The question arises whether these equations take a local form when written in coordinate space. This issue becomes important for gauge theories, where the lattice regulated coordinate space representation appears most natural. In order to answer this question, it is necessary to consider the momentum dependence of the kernels $\mathcal{M}^{(i)}(k, \omega)$ in (51). In the extreme infrared limit $k_{c} \rightarrow 0$ only the term $\mathcal{M}^{(c)}$ survives. The momentum dependence of this damping coefficient $\eta^{(c)}$ has been analyzed by Jeon [36] and by Wang and Heinz [37], who found that it varies only weakly over the range $0 \leq|\mathbf{k}|<m^{*}$. Outside that range, however, a strong momentum dependence $\sim 1 /|\mathbf{k}|$ is seen. A complete analysis of the two other damping coefficients $\eta^{(d)}$ and $\eta^{(e)}$ has not yet been performed, but a strong momentum dependence appears likely. On the other hand, these terms are subdominant in the infrared domain. Given that $E_{k}$ is also approaching a constant for $|\mathbf{k}| \ll m^{*}$, it may be possible to obtain a quasi-local form of the equation for the soft modes at distances larger than $1 / \mathrm{m}^{*}$. Clearly, a more complete analysis is required, also with regards to the important issue whether the noise terms can be approximated as local white noise, before a detailed understanding of thermalization of soft modes in quantum field theory is achieved. We hope to address these questions, as well as the issue of the numerical implementation of the equations derived here, in our future work.

\section{NOTE ADDED}

When finishing the manuscript the authors became aware of a recent manuscript by Boyanovsky, Lawrie and Lee on "Relaxation and Kinetics in Scalar Field Theories" [54, where a linearized equation of motion for the ensemble averaged field $\langle\Phi(\mathbf{x}, t)\rangle$ is derived containing the contribution (a) and (c) of the real part of the effective action (cf. Fig. 1). Their stated damping rate coincides with our result (61).

\section{ACKNOWLEDGMENTS}

We acknowledge stimulating discussions with M. Thoma. C.G. thanks the Alexander von Humboldt Stiftung for its partial support with a Feodor Lynen scholarship and also acknowledges support by the BMBF and GSI Darmstadt. This work was supported in part by the U.S. Department of Energy under grants DE-FG05-90ER40592 and DEFG02-96ER40945. 


\section{APPENDIX A: SHORT REVIEW OF THE INFLUENCE FUNCTIONAL TECHNIQUE (FEYNMAN)}

A quantum mechanical system $\mathrm{X}$ (described by the variable $x$ ) interacts with a bath $Q$ (described by the variable $q)$. At some specified initial time $t=t_{i}$ the combined system $(\mathrm{X} \cup \mathrm{Q})$ is described by the full density matrix

$$
\rho_{\mathrm{X} \cup \mathrm{Q}}\left(t_{i}\right)=\rho_{\mathrm{X} \cup \mathrm{Q}}\left(x_{i}, q_{i}, x_{i}^{\prime}, q_{i}^{\prime} ; t_{i}\right) .
$$

In the Schrödinger representation the (full) density matrix evolves in time according to

$$
\begin{aligned}
& \rho_{\mathrm{X} \cup \mathrm{Q}}\left(x, q ; x^{\prime}, q^{\prime} ; t\right) \\
& \quad=\int d x_{i} d q_{i} d x_{i}^{\prime} d q_{i}^{\prime}\left\{U\left(x, q ; x_{i}, q_{i} ; t, t_{i}\right) \rho_{\mathrm{X} \cup \mathrm{Q}}\left(x_{i}, q_{i} ; x_{i}^{\prime}, q_{i}^{\prime} ; t_{i}\right) U^{\dagger}\left(x_{i}^{\prime}, q_{i}^{\prime} ; x^{\prime}, q^{\prime} ; t, t_{i}\right)\right\}
\end{aligned}
$$

where the evolution operator reads in the path integral representation

$$
U\left(x, q ; x_{i}, q_{i} ; t, t_{i}\right)=\int_{x_{i}}^{x} \mathcal{D} x \int_{q_{i}}^{q} \mathcal{D} q e^{\frac{i}{\hbar} S[x, q]}
$$

with

$$
S[x, q]=\int_{t_{i}}^{t} d s \mathcal{L}(x(s), q(s))
$$

By formally integrating out the bath degrees of freedom $q$ one obtains an effective interaction $S_{\text {eff }}\left[x(s), x^{\prime}(s)\right]$ describing the evolution of the system degrees of freedom $x(s)$. This leads naturally to the "doubling" of the degrees of freedom in a real time description.

Typically one assumes that the initial density matrix $\rho_{\mathrm{X} \cup \mathrm{Q}}\left(t_{i}\right)$ is uncorrelated in its variables $x$ and $q$, i.e.

$$
\rho_{\mathrm{X} \cup \mathrm{Q}}\left(t_{i}\right)=\rho_{\mathrm{X}}\left(x_{i}, x_{i}^{\prime}, t_{i}\right) \otimes \rho_{\mathrm{Q}}\left(q_{i}, q_{i}^{\prime} ; t_{i}\right),
$$

which can be motivated by assuming that the interaction $\mathcal{L}_{\text {int }}(x, q)$ is adiabatically switched on at the time $t_{i}$.

Introducing the reduced density matrix $\rho_{r}$ as

$$
\rho_{r}\left(x, x^{\prime} ; t\right)=\operatorname{Tr}_{(q)}\left\{\rho\left(x, q ; x^{\prime}, q^{\prime} ; t\right)\right\} \equiv \rho_{\mathrm{X}}\left(x, x^{\prime} ; t\right)
$$

one finds that the expectation value of any operator $\hat{A}$ depending solely on the system degrees of freedom can be readily expressed in terms of $\rho_{r}$ :

$$
\langle A\rangle=\operatorname{Tr}_{(x, q)}\left\{A\left(x, x^{\prime} ; t\right) \rho_{\mathrm{X} \cup \mathrm{Q}}\left(x, q ; x^{\prime}, q^{\prime} ; t\right)\right\}=\operatorname{Tr}_{(x)}\left\{A\left(x, x^{\prime} ; t\right) \rho_{r}\left(x, x^{\prime} ; t\right)\right\}
$$

Moreover one finds that its evolution in time can be put in the general form

$$
\rho_{r}\left(x, x^{\prime} ; t\right)=\int d x_{i} d x_{i}^{\prime} \int_{x_{i}}^{x} \mathcal{D} x \int_{x_{i}^{\prime}}^{x^{\prime}} \mathcal{D} x^{\prime} e^{\frac{i}{\hbar}\left(S_{x}[x]-S_{x}\left[x^{\prime}\right]\right)} e^{\frac{i}{\hbar} S_{\mathrm{IF}}\left[x, x^{\prime}\right]} \rho_{r}\left(x_{i}, x_{i}^{\prime} ; t_{i}\right)
$$

by introducing the influence functional $S_{\mathrm{IF}}\left[x, x^{\prime}\right]$ [26]

$$
\begin{aligned}
e^{\frac{i}{\hbar} S_{\mathrm{IF}}\left[x \cdot x^{\prime}\right]} & =\operatorname{Tr}_{(q)}\left\{U_{Q}^{(x)}\left(q, q_{i} ; t, t_{i}\right) \rho_{Q}\left(q_{i}, q_{i}^{\prime} ; t_{i}\right) U_{Q}^{\left(x^{\prime}\right) \dagger}\left(q_{i}^{\prime}, q_{i} ; t, t_{i}\right)\right\} \\
& =\int d q d q_{i} d q_{i}^{\prime} \int_{q_{i}}^{q} \mathcal{D} q \int_{q_{i}^{\prime}}^{q} \mathcal{D} q^{\prime} e^{\frac{i}{\hbar}\left(S_{Q}[q]-S_{Q}\left[q^{\prime}\right]+S_{\mathrm{int}}[x, q]-S_{\mathrm{int}}\left[x^{\prime}, q^{\prime}\right]\right)} \rho_{Q}\left(q_{i}, q_{i}^{\prime} ; t_{i}\right) .
\end{aligned}
$$

Here $x(s), x^{\prime}(s)$ are treated as given, classical background fields. Thus one is led to say that $\rho_{r}$ evolves in time according to the effective interaction

$$
S_{\mathrm{eff}}\left[x, x^{\prime}\right]=S_{x}[x]-S_{x}\left[x^{\prime}\right]+S_{\mathrm{IF}}\left[x, x^{\prime}\right]
$$

It is clear that $\rho_{r}(t)$ evolves causally from the history of the system and the bath.

A notational simplification is achieved by introducing combined variables $x_{c}(s), q_{c}(s)$ defined on the real time contour $\mathcal{C}$ in the real time Green's function approach to finite temperature quantum field theory or nonequilibrium quantum field theory, i.e. 


$$
x_{c}(s)= \begin{cases}x(s) & s \in \text { upper branch } \\ x^{\prime}(s) & s \in \text { lower branch, }\end{cases}
$$

and similarly for $q(s), q\left(s^{\prime}\right)$, as illustrated in Figure A1. The influence then takes the more compact form

$$
e^{\frac{i}{\hbar} S_{\mathrm{IF}}\left[x_{c}\right]}=\int d q d q_{i} d q_{i}^{\prime} \int_{q_{i}}^{q} \mathcal{D} q \int_{q_{i}^{\prime}}^{q} \mathcal{D} q^{\prime} e^{\frac{i}{\hbar} \int{ }^{\mathcal{C}} d s_{c}\left\{\mathcal{L}_{Q}\left(q_{c}(s)\right)+\mathcal{L}_{\mathrm{int}}\left(x_{c}(s), q_{c}(s)\right)\right\}} \rho_{Q}\left(q_{i}, q_{i}^{\prime} ; t_{i}\right),
$$

where the integration is defined on the time contour from $t_{i}$ forward to $t$ and back to $t_{i}$.

For the influence action $S_{\mathrm{IF}}\left[x, x^{\prime}\right]$ one finds the following general properties to hold:

$$
\begin{aligned}
S_{\mathrm{IF}}\left[x, x^{\prime}\right] & =-\left(S_{\mathrm{IF}}\left[x^{\prime}, x\right]\right)^{*}, \\
S_{\mathrm{IF}}[x, x] & =0 .
\end{aligned}
$$

Expanding $S_{\mathrm{IF}}$ up to second order in $x$ and $x^{\prime}$, the general structure of $S_{\mathrm{IF}}$ is given by [26]

$$
\begin{aligned}
S_{\mathrm{IF}}\left[x, x^{\prime}\right] & \approx \int_{t_{i}}^{t} d s F(s)\left(x(s)-x^{\prime}(s)\right) \\
& +\frac{1}{2} \int_{t_{i}}^{t} d s_{1} d s_{2}\left(x\left(s_{1}\right)-x^{\prime}\left(s_{1}\right)\right) R\left(s_{1}, s_{2}\right)\left(x\left(s_{2}\right)+x^{\prime}\left(s_{2}\right)\right) \\
& +\frac{i}{2} \int_{t_{i}}^{t} d s_{1} d s_{2}\left(x\left(s_{1}\right)-x^{\prime}\left(s_{1}\right)\right) I\left(s_{1}, s_{2}\right)\left(x\left(s_{2}\right)-x^{\prime}\left(s_{2}\right)\right) .
\end{aligned}
$$

Suppose that $\mathcal{L}_{\text {int }}$ takes the quite general form

$$
\mathcal{L}_{\text {int }}\left[x_{c}, q_{c}\right]=f\left(x_{c}(s)\right) \Xi\left(q_{c}(s)\right) .
$$

Writing for $S_{\mathrm{IF}}[x]$ up to second order in $f$

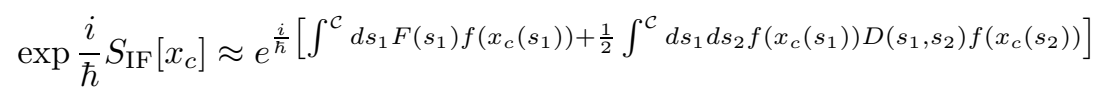

it follows by comparing with (A12) and performing the substitution $x(s) \rightarrow f(x(s))$

$$
\begin{aligned}
F\left(s_{1}\right) & =\left(\frac{\hbar}{i}\right) \frac{\delta e^{\frac{i}{\hbar} S_{\mathrm{IF}}}}{\delta f\left(x_{c}\left(s_{1}\right)\right)}=\left\langle\Xi\left(q_{c}\left(s_{1}\right)\right)\right\rangle_{\rho_{Q}} \\
D\left(s_{1}, s_{2}\right) & =\left(\frac{\hbar}{i}\right) \frac{\delta^{2} e^{\frac{i}{\hbar} S_{\mathrm{IF}}}}{\delta f\left(x_{c}\left(s_{1}\right)\right) \delta f\left(x_{1}\left(s_{2}\right)\right)} \\
& =\frac{i}{\hbar}\left[\left\langle P\left(\Xi\left(q_{c}\left(s_{1}\right)\right) \Xi\left(q_{c}\left(s_{2}\right)\right)\right)\right\rangle-\left\langle\Xi\left(q_{c}\left(s_{1}\right)\right)\right\rangle\left\langle\Xi\left(q_{c}\left(s_{2}\right)\right)\right\rangle\right]
\end{aligned}
$$

where $P$ means path-ordering along the contour $\mathcal{C}$. $F(s)$ can be interpreted as an external force term due to the mean field generated by the average interaction of the bath variables $Q$ with the system $X$.

The path-ordering definition leads to the typical four Green's functions defined in real time:

$$
\begin{aligned}
D^{++}\left(t_{1}, t_{2}\right) & =\frac{i}{\hbar}\left(\left\langle T\left(\Xi\left(q\left(t_{1}\right)\right) \Xi\left(q\left(t_{2}\right)\right)\right\rangle-\left\langle\Xi\left(q\left(t_{1}\right)\right)\right\rangle\left\langle\Xi\left(q\left(t_{2}\right)\right)\right\rangle\right)\right. \\
& =\theta\left(t_{1}-t_{2}\right) D^{-+}\left(t_{1}, t_{2}\right)+\theta\left(t_{2}-t_{1}\right) D^{+-}\left(t_{1}, t_{2}\right) \\
D^{+-}\left(t_{1}, t_{2}\right) & =\frac{i}{\hbar}\left(\left\langle\Xi\left(q\left(t_{2}\right)\right) \Xi\left(q\left(t_{1}\right)\right)\right\rangle-\left\langle\Xi\left(q\left(t_{1}\right)\right)\right\rangle\left\langle\Xi\left(q\left(t_{2}\right)\right)\right\rangle\right) \equiv D^{<}\left(t_{1}, t_{2}\right) \\
D^{-+}\left(t_{1}, t_{2}\right) & =\frac{i}{\hbar}\left(\left\langle\Xi\left(q\left(t_{1}\right)\right) \Xi\left(q\left(t_{2}\right)\right)\right\rangle-\left\langle\Xi\left(q\left(t_{1}\right)\right)\right\rangle\left\langle\Xi\left(q\left(t_{2}\right)\right)\right\rangle\right) \equiv D^{>}\left(t_{1}, t_{2}\right) \\
D^{--}\left(t_{1}, t_{2}\right) & =\frac{i}{\hbar}\left(\left\langle\tilde{T}\left(\Xi\left(q\left(t_{1}\right)\right) \Xi\left(q\left(t_{2}\right)\right)\right\rangle-\left\langle\Xi\left(q\left(t_{1}\right)\right)\right\rangle\left\langle\Xi\left(q\left(t_{2}\right)\right)\right\rangle\right)\right. \\
& =\theta\left(t_{1}-t_{2}\right) D^{+-}\left(t_{1}, t_{2}\right)+\theta\left(t_{2}-t_{1}\right) D^{-+}\left(t_{2}, t_{2}\right)
\end{aligned}
$$

Here $T(\tilde{T})$ stands for (anti)-time ordering. 
Defining the real Green's functions

$$
\begin{aligned}
D_{R}\left(t_{1}, t_{2}\right) & =D^{>}\left(t_{1}, t_{2}\right)-D^{<}\left(t_{1}, t_{2}\right)=\frac{i}{\hbar}\left\langle\left[\Xi\left(q\left(t_{1}\right)\right), \Xi\left(q\left(t_{2}\right)\right)\right]\right\rangle \\
D_{I}\left(t_{1}, t_{2}\right) & =\frac{1}{i}\left(D^{>}\left(t_{1}, t_{2}\right)+D^{<}\left(t_{1}, t_{2}\right)\right) \\
& \left.=\frac{1}{\hbar}\left(\left\langle\{\Xi(q(t) 1)), \Xi\left(q\left(t_{2}\right)\right)\right\}\right\rangle-2\left\langle\Xi\left(q\left(t_{1}\right)\right)\right\rangle\left\langle\Xi\left(q\left(t_{2}\right)\right)\right\rangle\right),
\end{aligned}
$$

where $D_{R}\left(t_{1}, t_{2}\right)=-D_{R}\left(t_{2}, t_{1}\right)$ and $D_{I}\left(t_{1}, t_{2}\right)=D_{I}\left(t_{2}, t_{1}\right)$, one finds after some algebra that in (A15) $R$ and $I$ are given by

$$
\begin{aligned}
R\left(t_{1}, t_{2}\right) & =D_{R}\left(t_{1}, t_{2}\right) \theta\left(t_{1}-t_{2}\right)=2 \operatorname{Re}\left(D^{++}\left(t_{1}, t_{2}\right)\right) \theta\left(t_{1}-t_{2}\right) \\
I\left(t_{1}, t_{2}\right) & =\frac{1}{2} D_{I}\left(t_{1}, t_{2}\right)=\operatorname{Im}\left(D^{++}\left(t_{1}, t_{2}\right)\right) .
\end{aligned}
$$

If the system behaves quasi-classically, the density matrix $\rho_{r}$ becomes nearly diagonal. Then from (A\&), the major paths contributing to the evolution of the reduced density matrix are obtained by extremizing the effective action $S_{\mathrm{eff}}\left[x, x^{\prime}\right]$ :

$$
x(s)=x^{\prime}(s) \text { for all } t_{i} \leq s<t
$$

and the extremity conditions

$$
\left.\frac{\delta S_{\mathrm{eff}}\left[x, x^{\prime}\right]}{\delta x(s)}\right|_{x=x^{\prime}}=0
$$

The equations of motion are clearly causal if one constructs the effective action to the argument $s=t$.

Typically one introduces the variables

$$
\bar{x}=\frac{x+x^{\prime}}{2}, \quad \Delta=x-x^{\prime}
$$

so that the semiclassical equations of motion A23 for $\bar{x}$ are stated as

$$
\left.\frac{\delta S_{\mathrm{eff}}(\bar{x}, \Delta)}{\delta \Delta(s)}\right|_{\Delta=0}=0
$$

From A15 we have by expansion up to second order

$$
\frac{i}{\hbar} S_{\mathrm{eff}}(\bar{x}, \Delta)=\frac{i}{\hbar} \Delta \otimes\left(\frac{\delta S_{X}[\bar{x}]}{\delta \bar{x}(s)}+F(s)+R \otimes \bar{x}\right)-\frac{1}{2 \hbar} \Delta \otimes I \otimes \Delta,
$$

thus

$$
-\frac{\delta S_{X}[\bar{x}]}{\delta \bar{x}(s)}-F(s)-\int_{t_{i}}^{s} d s^{\prime} R\left(s, s^{\prime}\right) \bar{x}\left(s^{\prime}\right)=0
$$

as the average quasi-classical equation of motion. This equation, in return, has to be interpreted as an average over random, fluctuating forces. The last contribution in (A26) leads to decoherence because any path contributing with sizeable $|\Delta|>0$ over past time becomes exponentially suppressed-hence, the imaginary part $I$ drives the system to quasi-classical behavior. For short periods in time, however, fluctuations in $\Delta$ can appear on the order of $\left|\int d t_{1} d t_{2} I\left(t_{1}, t_{2}\right)\right|^{-1 / 2}$ stochastically. These act as random "kicks" on the actual trajectory and can be interpreted as a stochastic force [17]. To see this in more detail, one defines the real stochastic influence action

$$
\tilde{S}_{\mathrm{IF}}\left[x, x^{\prime}, \xi\right]=\operatorname{Re}\left(S_{\mathrm{IF}}\left[x, x^{\prime}\right]\right)+\int_{t_{i}}^{t} d s \xi(s)\left(x(s)-x^{\prime}(s)\right),
$$

where $\xi(s)$ is interpreted as an external force, randomly distributed by a Gaussian distribution with zero mean:

$$
P[\xi(s)]=\frac{1}{N} \exp \left(-\frac{1}{2 \hbar} \int_{t_{i}}^{t} d s_{1} d s_{2} \xi\left(s_{1}\right) I^{-1}\left(s_{1}, s_{2}\right) \xi\left(s_{2}\right)\right) .
$$


The influence functional $S_{\mathrm{IF}}\left[x, x^{\prime}\right]$ is regained as the characteristic functional over the average of the random forces.

$$
e^{i S_{\mathrm{IF}}\left[x, x^{\prime}\right]} \equiv \phi_{\xi}\left[x(s)-x^{\prime}(s)\right]=\int D \xi P[\xi(s)] e^{i \tilde{S}_{\mathrm{IF}}\left[x, x^{\prime}, \xi\right]} .
$$

The imaginary part $I$ also defines the correlation of the random forces

$$
\left\langle\xi\left(s_{1}\right) \xi\left(s_{2}\right)\right\rangle_{\xi}=\hbar I\left(s_{1}, s_{2}\right) .
$$

From (A28) the corresponding equation of motion reads

$$
-\frac{\delta S_{X}[\bar{x}]}{\delta \bar{x}(s)}-F(s)-\int_{t_{i}}^{s} d s^{\prime} R\left(s, s^{\prime}\right) \bar{x}\left(s^{\prime}\right)=\xi(s)
$$

as the effective Langevin like equation dictating the dynamics of the system variable in the quasi-classical regime. (Classical) Brownian Motion [17] is recovered if the spectral function of the system allows for approximating the kernels $R$ and $I$ as

$$
\begin{aligned}
R\left(s, s^{\prime}\right) & =-m \gamma \dot{\delta}\left(s, s^{\prime}\right), \\
\hbar I\left(s, s^{\prime}\right) & =2 m \gamma k T \delta\left(s, s^{\prime}\right) .
\end{aligned}
$$

In summary, by coarse-graining over the bath degrees of freedom, there appear two major contributions to the evolution of the reduced density matrix (A8 A26), namely the oscillatory or quantum mechanical phase and the decoherence factor when summing over paths in the $(\bar{x}-\Delta)$-plane. We do expect an essentially semiclassical evolution when the excitation energy of the system (divided by $\hbar$ ) is large so that the oscillatory phases of paths neighboring the classical path interferes destructively. Moreover, all paths where the time averaged $\Delta$ is sufficiently large will be exponentially suppressed by the decoherence factor. Hence, the system will be driven by decoherence into the quasiclassical regime, depending crucially on the magnitude $\sqrt{\left\langle\xi^{2}\right\rangle} \sim \sqrt{I}$ of the random forces, i.e. the size of the noise kernel (A31). If it is large, decoherence should happen very rapidly, and the quasi-classical description is inherently stochastic.

\section{APPENDIX B: THE INFLUENCE ACTION FOR SCALAR $\phi^{4}$ THEORY}

Dividing the scalar field $\Phi$ into soft modes $\phi$ and hard modes $\varphi$, the completely analogous expression for the influence functional can be read off (A12):

$$
e^{\frac{i}{\hbar} S_{\mathrm{IF}}\left[\phi, \phi^{\prime}\right]}=\int d \varphi \int_{\varphi_{i}}^{\varphi} \mathcal{D} \varphi \int_{\varphi_{i}^{\prime}}^{\varphi} \mathcal{D} \varphi^{\prime} e^{\frac{i}{\hbar} \int^{\mathcal{C}} d^{4} s\left[\mathcal{L}_{h}^{0}[\varphi]+\mathcal{L}_{\mathrm{int}}^{\prime}[\phi, \varphi]\right]} \rho_{h}\left(\varphi_{i}, \varphi_{i}^{\prime} ; t_{i}\right),
$$

where in the definition of $S_{\mathrm{int}}^{\prime}[\phi, \varphi]$

$$
S_{\mathrm{int}}^{\prime}[\phi, \varphi]=g^{2} \int_{t_{i}}^{t} d^{4} x\left[-\frac{1}{24} \varphi^{4}-\frac{1}{6} \phi \varphi^{3}-\frac{1}{4} \phi^{2} \varphi^{2}-\frac{1}{6} \phi^{3} \varphi\right]
$$

also the self interaction of the hard modes is included and shall be treated perturbatively.

Expanding the influence functional up to order $g^{4}$,

$$
S_{\mathrm{IF}}=S_{\mathrm{IF}}^{(0)}+S_{\mathrm{IF}}^{(1)}+S_{\mathrm{IF}}^{(2)},
$$

one finds in an obvious notation:

$$
\begin{aligned}
& S_{\mathrm{IF}}^{(0)}=1, \\
& S_{\mathrm{IF}}^{(1)} \equiv\left\langle\int^{\mathcal{C}} d^{4} s \mathcal{L}_{\mathrm{int}}^{\prime}[\phi, \varphi]\right\rangle_{0} \equiv \int d \varphi \int_{\varphi_{i}}^{\varphi} \mathcal{D} \varphi \int_{\varphi_{i}}^{\varphi} \mathcal{D} \varphi^{\prime}\left(\int^{\mathcal{C}} d^{4} s \mathcal{L}_{\mathrm{int}}^{\prime}\right) e^{\frac{i}{\hbar} \int^{\mathcal{C}} d^{4} s \mathcal{L}_{h}^{0}[\varphi]} \rho_{h}\left(\varphi_{i}, \varphi_{i}^{\prime} ; t_{i}\right) \\
& S_{\mathrm{IF}}^{(2)}=\frac{1}{2} \frac{i}{\hbar}\left(\left\langle\int^{\mathcal{C}} d^{4} s_{1} d^{4} s_{2} P\left(\mathcal{L}_{\mathrm{int}}^{\prime}\left(\phi\left(s_{1}\right), \varphi\left(s_{1}\right)\right) \mathcal{L}_{\mathrm{int}}^{\prime}\left(\phi\left(s_{2}\right), \varphi\left(s_{2}\right)\right)\right)\right\rangle_{0}-\left(S_{\mathrm{IF}}^{(1)}\right)^{2}\right)
\end{aligned}
$$


The expectation values can be evaluated perturbatively with standard real time Green's function techniques [52,53]. To avoid difficulties with initial higher order correlations of $\rho_{h}\left(t_{i}\right)$ one typically has to assume that $\rho_{h}\left(t_{i}\right)$ is of the form

$$
\rho_{Q ; 0} \equiv \rho_{h}\left(t_{i}\right)=\exp \left(\sum_{p} A_{p} \hat{a}_{p}^{\dagger} \hat{a}_{p}\right), \quad\left(A_{p}<0\right)
$$

to allow for a simple Wick decomposition. A thermal distribution of non-interacting hard particles is thus appropriate. We define the real time propagator of the hard particles on the contour as

$$
G_{c}^{k_{c}}\left(x_{1}, x_{2}\right)=i\left\langle P\left(\varphi\left(x_{1}\right) \varphi\left(x_{2}\right)\right)\right\rangle_{0}
$$

with its components in real time

$$
\begin{aligned}
& G_{++}^{k_{c}}\left(x_{1}, x_{2}\right)=i\left\langle T\left(\varphi\left(x_{1}\right) \varphi\left(x_{2}\right)\right)\right\rangle_{0}=\theta\left(t_{1}-t_{2}\right) G_{-+}^{k_{c}}\left(x_{1}, x_{2}\right)+\theta\left(t_{2}-t_{1}\right) G_{+-}^{k_{c}}\left(x_{1}, x_{2}\right) \\
& G_{+-}^{k_{c}}\left(x_{1}, x_{2}\right)=i\left\langle\varphi\left(x_{2}\right) \varphi\left(x_{1}\right)\right\rangle_{0} \equiv G_{<}^{k_{c}}\left(x_{1}, x_{2}\right) \\
& G_{-+}^{k_{c}}\left(x_{1}, x_{2}\right)=i\left\langle\varphi\left(x_{1}\right) \varphi\left(x_{2}\right)\right\rangle_{0} \equiv G_{>}^{k_{c}}\left(x_{1}, x_{2}\right) \\
& G_{--}^{k_{c}}\left(x_{1}, x_{2}\right)=i\left\langle\tilde{T}\left(\varphi\left(x_{1}\right) \varphi\left(x_{2}\right)\right)\right\rangle_{0},
\end{aligned}
$$

which have a simple Fourier representation

$$
\begin{aligned}
& G_{>}^{k_{c}}(\mathbf{p}, \tau)=G_{<}^{k_{c}}(\mathbf{p},-\tau)=i \frac{\left[1+2 n\left(\omega_{p}\right)\right] \cos \omega_{p} \tau-i \sin \omega_{p} \tau}{2 \omega_{p}} \text { for }|p| \geq k_{c} \\
& G_{>}^{k_{c}}(\mathbf{p}, \tau)=G_{<}^{k_{c}}(\mathbf{p},-\tau)=0 \text { for }|p|<k_{c}
\end{aligned}
$$

The evaluation of the expectation values in (B4) is lengthy but straightforward. We state only the final results with their real and imaginary parts:

$$
\begin{aligned}
S_{\mathrm{IF}}^{(1 a)}= & i \frac{g^{2}}{4} \int^{\mathcal{C}} d^{4} x G_{c}^{k_{c}}(x, x) \phi(x)^{2} \\
= & i \frac{g^{2}}{4} \int_{t_{i}}^{t} d^{4} x\left(\phi(x)-\phi^{\prime}(x)\right) G_{>}^{k_{c}}(0)\left(\phi(x)+\phi^{\prime}(x)\right) \\
S_{\mathrm{IF}}^{(2 b)}=- & \frac{g^{4}}{8} \int^{\mathcal{C}} d^{4} x_{1} d^{4} x_{2} \phi\left(x_{1}\right)^{2}\left(G_{c}^{k_{c}}\left(x_{1}, x_{2}\right)\right)^{2} G_{c}^{k_{c}}\left(x_{2}, x_{2}\right) \\
=- & \frac{g^{4}}{8} \int_{t_{i}}^{t} d^{4} x_{1} d^{4} x_{2}\left(\phi\left(x_{1}\right)-\phi^{\prime}\left(x_{1}\right)\right) \\
& \times\left\{\theta\left(t_{1}-t_{2}\right)\left(G_{>}^{k_{c}}\left(x_{1}, x_{2}\right)^{2}-G_{<}^{k_{c}}\left(x_{1}, x_{2}\right)^{2}\right) G_{>}^{k_{c}}(0)\right\}\left(\phi\left(x_{1}\right)+\phi^{\prime}\left(x_{1}\right)\right) \\
S_{\mathrm{IF}}^{(2 c)}=- & \frac{g^{4}}{12} \int^{\mathcal{C}} d^{4} x_{1} d^{4} x_{2} \phi\left(x_{1}\right)\left(G_{c}^{k_{c}}\left(x_{1}, x_{2}\right)\right)^{3} \phi\left(x_{2}\right) \\
=- & \frac{g^{4}}{12} \int_{t_{i}}^{t} d^{4} x_{1} d^{4} x_{2}\left\{\left(\phi\left(x_{1}\right)-\phi^{\prime}\left(x_{1}\right)\right) \theta\left(t_{1}-t_{2}\right)\right. \\
& \left.\times\left(G_{>}^{k_{c}}\left(x_{1}, x_{2}\right)^{3}-G_{<}^{k_{c}}\left(x_{1}, x_{2}\right)^{3}\right)\left(\phi\left(x_{2}\right)+\phi^{\prime}\left(x_{2}\right)\right)\right\} \\
& -i \frac{g^{4}}{12} \int_{t_{i}}^{t} d^{4} x_{1} d^{4} x_{2}\left\{\left(\phi\left(x_{1}\right)-\phi^{\prime}\left(x_{1}\right)\right)\right. \\
& \left.\times \frac{1}{2 i}\left(G_{>}^{k_{c}}\left(x_{1}, x_{2}\right)^{3}+G_{<}^{k_{c}}\left(x_{1}, x_{2}\right)^{3}\right)\left(\phi\left(x_{2}\right)-\phi^{\prime}\left(x_{2}\right)\right)\right\} \\
S_{\mathrm{IF}}^{(2 d)}=- & i \frac{g^{4}}{16} \int^{\mathcal{C}} d^{4} x_{1} d^{4} x_{2} \phi\left(x_{1}\right)^{2}\left(G_{c}^{k_{c}}\left(x_{1}, x_{2}\right)\right)^{2} \phi\left(x_{2}\right)^{2} \\
= & i \frac{g^{4}}{16} \int_{t_{i}}^{t} d^{4} x_{1} d^{4} x_{2}\left\{\left(\phi\left(x_{1}\right)^{2}-\phi^{\prime}\left(x_{1}\right)^{2}\right) \theta\left(t_{1}-t_{2}\right)\right. \\
& \\
& \\
&
\end{aligned}
$$




$$
\begin{aligned}
&\left.\times\left(G_{>}^{k_{c}}\left(x_{1}, x_{2}\right)^{2}-G_{<}^{k_{c}}\left(x_{1}, x_{2}\right)^{2}\right)\left(\phi\left(x_{2}\right)^{2}+\phi^{\prime}\left(x_{2}\right)^{2}\right)\right\} \\
&-i \frac{g^{4}}{16} \int_{t_{i}}^{t} d^{4} x_{1} d^{4} x_{2}\left\{\left(\phi\left(x_{1}\right)^{2}-\phi^{\prime}\left(x_{1}\right)^{2}\right)\right. \\
&\left.\times \frac{1}{2}\left(G_{>}^{k_{c}}\left(x_{1}, x_{2}\right)^{2}+G_{<}^{k_{c}}\left(x_{1}, x_{2}\right)^{2}\right)\left(\phi\left(x_{2}\right)^{2}-\phi^{\prime}\left(x_{2}\right)^{2}\right)\right\} \\
& S_{\mathrm{IF}}^{(2 e)}=\frac{g^{4}}{72} \int^{\mathcal{C}} d^{4} x_{1} d^{4} x_{2} \phi\left(x_{1}\right)^{3} G_{c}^{k_{c}}\left(x_{1}, x_{2}\right) \phi\left(x_{2}\right)^{3} \\
&=\frac{g^{4}}{72} \int_{t_{i}}^{t} d^{4} x_{1} d^{4} x_{2}\left\{\left(\phi\left(x_{1}\right)^{3}-\phi^{\prime}\left(x_{1}\right)^{3}\right) \theta\left(t_{1}-t_{2}\right)\right. \\
&\left.\quad \times\left(G_{>}^{k_{c}}\left(x_{1}, x_{2}\right)-G_{<}^{k_{c}}\left(x_{1}, x_{2}\right)\right)\left(\phi\left(x_{2}\right)^{3}+\phi^{\prime}\left(x_{2}\right)^{3}\right)\right\} \\
&+i \frac{g^{4}}{72} \int_{t_{i}}^{t} d^{4} x_{1} d^{4} x_{2}\left\{\left(\phi\left(x_{1}\right)^{3}-\phi^{\prime}\left(x_{1}\right)^{3}\right)\right. \\
&\left.\quad \times \frac{1}{2 i}\left(G_{>}^{k_{c}}\left(x_{1}, x_{2}\right)+G_{<}^{k_{c}}\left(x_{1}, x_{2}\right)\right)\left(\phi\left(x_{2}\right)^{3}-\phi^{\prime}\left(x_{2}\right)^{3}\right)\right\}
\end{aligned}
$$

Momentum space expressions can be found by inserting (B7) into these equations. Introducing the new field variables

$$
\bar{\phi}=\frac{1}{2}\left(\phi+\phi^{\prime}\right), \quad \phi_{\Delta}=\phi-\phi^{\prime}
$$

and using the relations

$$
\begin{aligned}
& \operatorname{Re}\left(G_{++}^{k_{c}}\left(x_{1}, x_{2}\right)^{n}\right)=\left(\theta\left(t_{1}-t_{2}\right)+(-1)^{n} \theta\left(t_{2}-t_{1}\right)\right) \frac{1}{2}\left(G_{>}^{k_{c}}\left(x_{1}, x_{2}\right)^{n}+(-1)^{n} G_{<}^{k_{c}}\left(x_{1}, x_{2}\right)^{n}\right) \\
& \operatorname{Im}\left(G_{++}^{k_{c}}\left(x_{1}, x_{2}\right)^{n}\right)=\left(\theta\left(t_{1}-t_{2}\right)-(-1)^{n} \theta\left(t_{2}-t_{1}\right)\right) \frac{1}{2 i}\left(G_{>}^{k_{c}}\left(x_{1}, x_{2}\right)^{n}-(-1)^{n} G_{<}^{k_{c}}\left(x_{1}, x_{2}\right)^{n}\right)
\end{aligned}
$$

one finds after some calculation for the real and imaginary part, respectively, of the influence action

$$
\begin{aligned}
\operatorname{Re} S_{\mathrm{IF}} & {\left[\phi_{\Delta}, \bar{\phi}\right]=-\frac{g^{2}}{2} \int_{t_{i}}^{t} d^{4} x_{1} \phi_{\Delta}\left(x_{1}\right) \bar{\phi}\left(x_{1}\right) \operatorname{Im}\left[G_{++}^{k_{c}}(0)\right] } \\
& +g^{4} \int_{t_{i}}^{t} d^{4} x_{1} d^{4} x_{2} \theta\left(t_{1}-t_{2}\right)\left\{\frac{1}{2} \phi_{\Delta}\left(x_{1}\right) \bar{\phi}\left(x_{1}\right) \operatorname{Im} G_{++}^{k_{c}}(0) \operatorname{Im}\left[G_{++}^{k_{c}}\left(x_{1}, x_{2}\right)^{2}\right]\right. \\
& -\frac{1}{3} \phi_{\Delta}\left(x_{1}\right) \operatorname{Re}\left[G_{++}^{k_{c}}\left(x_{1}, x_{2}\right)^{3}\right] \bar{\phi}\left(x_{2}\right) \\
& +\frac{1}{2} \bar{\phi}\left(x_{1}\right) \phi_{\Delta}\left(x_{1}\right) \operatorname{Im}\left[G_{++}^{k_{c}}\left(x_{1}, x_{2}\right)^{2}\right]\left(\bar{\phi}\left(x_{2}\right)^{2}+\frac{1}{4} \phi_{\Delta}\left(x_{2}\right)^{2}\right) \\
& \left.+\frac{1}{6} \phi_{\Delta}\left(x_{1}\right)\left(\bar{\phi}^{2}\left(x_{1}\right)+\frac{1}{12} \phi_{\Delta}^{2}\left(x_{1}\right)\right) \operatorname{Re}\left[G_{++}^{k_{c}}\left(x_{1}, x_{2}\right)\right] \bar{\phi}\left(x_{2}\right)\left(\bar{\phi}\left(x_{2}\right)^{2}+\frac{3}{4} \phi_{\Delta}\left(x_{2}\right)^{2}\right)\right\},
\end{aligned}
$$

and

$$
\begin{aligned}
\operatorname{Im} S_{\mathrm{IF}}\left[\phi_{\Delta}, \bar{\phi}\right]=g^{4} & \int_{t_{i}}^{t} d^{4} x_{1} d^{4} x_{2}\left\{-\frac{1}{12} \phi_{\Delta}\left(x_{1}\right) \operatorname{Im}\left[G_{++}^{k_{c}}\left(x_{1}, x_{2}\right)^{3}\right] \phi_{\Delta}\left(x_{2}\right)\right. \\
& -\frac{1}{4} \phi_{\Delta}\left(x_{1}\right) \bar{\phi}\left(x_{1}\right) \operatorname{Re}\left[G_{++}^{k_{c}}\left(x_{1}, x_{2}\right)^{2}\right] \phi_{\Delta}\left(x_{2}\right) \bar{\phi}\left(x_{2}\right) \\
& +\frac{1}{8} \phi_{\Delta}\left(x_{1}\right)\left(\bar{\phi}\left(x_{1}\right)^{2}+\frac{1}{12} \phi_{\Delta}\left(x_{1}\right)^{2}\right) \\
& \times \operatorname{Im}\left[G_{++}^{k_{c}}\left(x_{1}, x_{2}\right)\right] \phi_{\Delta}\left(x_{2}\right)\left(\bar{\phi}\left(x_{2}\right)^{2}+\frac{1}{12} \phi_{\Delta}\left(x_{2}\right)^{2}\right) .
\end{aligned}
$$

After introducing the stochastic auxiliary functions $\xi_{i}(x), i=1,2,3$, the imaginary part of the influence action becomes 


$$
\begin{aligned}
\operatorname{Im} S_{\mathrm{IF}}\left[\phi_{\Delta}, \bar{\phi}\right] \rightarrow \tilde{S}_{\mathrm{IF}}\left[\bar{\phi}, \phi_{\Delta} ; \xi_{i}\right] \\
\quad=\int_{t_{i}}^{t} d^{4} x\left[\phi_{\Delta}(x) \xi_{1}(x)+\phi_{\Delta}(x) \bar{\phi}(x) \xi_{2}(x)+\phi_{\Delta}(x)\left(\bar{\phi}(x)^{2}+\frac{1}{12} \phi_{\Delta}(x)^{2}\right) \xi_{3}(x)\right]
\end{aligned}
$$

The corresponding stochastic weights are

$$
\begin{aligned}
& P\left[\xi_{1}\right]=N_{1}\left\{\int_{t_{i}}^{t} d^{4} x_{1} d^{4} x_{2} \xi_{1}\left(x_{1}\right) \frac{3}{g^{4}}\left[\operatorname{Im}\left(G_{++}^{k_{c}}\right)^{3}\right]_{x_{1}, x_{2}}^{-1} \xi_{1}\left(x_{2}\right)\right\} \\
& P\left[\xi_{2}\right]=N_{2}\left\{\int_{t_{i}}^{t} d^{4} x_{1} d^{4} x_{2} \xi_{2}\left(x_{1}\right) \frac{1}{g^{4}}\left[\operatorname{Re}\left(G_{++}^{k}\right)^{2}\right]_{x_{1}, x_{2}}^{-1} \xi_{2}\left(x_{2}\right)\right\} \\
& P\left[\xi_{3}\right]=N_{3}\left\{\int_{t_{i}}^{t} d^{4} x_{1} d^{4} x_{2} \xi_{3}\left(x_{1}\right)\left(\frac{-2}{g^{4}}\right)\left[\operatorname{Im}\left(G_{++}^{k_{c}}\right)\right]_{x_{1}, x_{2}}^{-1} \xi_{3}\left(x_{2}\right)\right\} .
\end{aligned}
$$

\section{APPENDIX C: DERIVATION OF TWO DAMPING COEFFICIENTS}

Following the interpretation developed in Section III.A, the dissipative contribution due to $\operatorname{Re}\left(S_{\mathrm{IF}}^{(d)}\right)$ to the equation of motion of the low momentum mode $\phi(\mathbf{k}, t)$ is stated as:

$$
\begin{aligned}
\int^{k_{c}} & \frac{d^{3} k_{2}}{(2 \pi)^{3}} \Upsilon_{\text {diss. }}^{(d)}\left(\mathbf{k}-\mathbf{k}_{2}, t\right) \phi\left(\mathbf{k}_{2}, t\right)=\int^{k_{c}} \frac{d^{3} k_{2}}{(2 \pi)^{3}} \Upsilon_{\text {diss. }}^{(d)}\left(\mathbf{k}+\mathbf{k}_{2}, t\right) \phi^{*}\left(\mathbf{k}_{2}, t\right) \\
= & \int^{k_{c}} \frac{d^{3} k_{1} d^{3} k_{2}}{(2 \pi)^{6}} \dot{\phi}\left(\mathbf{k}-\mathbf{k}_{1}+\mathbf{k}_{2}, t\right) \phi\left(\mathbf{k}_{1}, t\right) \phi^{*}\left(\mathbf{k}_{2}, t\right) \frac{1}{4 E_{\mathbf{k}-\mathbf{k}_{1}+\mathbf{k}_{2}}} \theta\left(k_{c}-\left|\mathbf{k}-\mathbf{k}_{1}+\mathbf{k}_{2}\right|\right) \\
& \times\left(i \mathcal{M}^{(d)}\left(\mathbf{k}+\mathbf{k}_{2}, E_{\mathbf{k}-\mathbf{k}_{1}+\mathbf{k}_{2}}+E_{k_{1}}\right)+i \mathcal{M}^{(d)}\left(\mathbf{k}+\mathbf{k}_{2}, E_{\mathbf{k}-\mathbf{k}_{1}+\mathbf{k}_{2}}-E_{k_{1}}\right)\right) .
\end{aligned}
$$

This is a highly nonlinear term where the integrand rapidly oscillates over the whole integration region. However, its 'major' contribution is expected for momenta $\mathbf{k}_{1} \approx \mathbf{k}_{2}$ because then $\phi\left(\mathbf{k}_{1}, t\right) \phi^{*}\left(\mathbf{k}_{2}, t\right) \approx\left|\phi\left(\mathbf{k}_{1}, t\right)\right|^{2}>0$. It is not our objective to show that all other contributions cancel, but to extract the dominant contribution to the integrals in (C1). Indeed, setting $\mathbf{k}_{1}=\mathbf{k}_{2}$ corresponds to the Wick contraction in standard thermal field theory. Suppose the above expression would be taken as an ensemble average over all possible and similar configurations, i.e. initial conditions, thus averaging over all the fluctuating phases. For a quasi-homogeneous system one will find for the correlator

$$
\left\langle\phi\left(\mathbf{k}_{1}, t\right) \phi^{*}\left(\mathbf{k}_{2}, t\right)\right\rangle \approx(2 \pi)^{3} \delta^{3}\left(\mathbf{k}_{2}-\mathbf{k}_{1}\right)\left|\phi\left(\mathbf{k}_{1}, t\right)\right|^{2} .
$$

Here

$$
\left|\phi\left(\mathbf{k}_{1}, t\right)\right|^{2} \approx \frac{1}{E_{k_{1}}} N\left(\mathbf{k}_{1}, t\right) \approx \frac{1}{E_{k_{1}}}\left(1+N\left(\mathbf{k}_{1}, t\right)\right)
$$

which is identified with the average population number in the classical regime, when the mode population is large. (C2) is then completely analogous to the equal-time limit of the propagators (defined in Appendix B) in a homogeneous system:

$$
-i G_{>}(\mathbf{k}, \tau=0) \approx n\left(\omega_{k}\right) / \omega_{k} .
$$

As a further motivation let us first calculate the classical vertex contribution to the equation of motion by means of the approximation ( 2 ):

$$
\begin{aligned}
& \frac{\tilde{g}^{2}}{3 !} \int^{k_{c}} \frac{d^{3} k_{1} d^{3} k_{2}}{(2 \pi)^{6}} \phi\left(\mathbf{k}-\mathbf{k}_{1}-\mathbf{k}_{2}, t\right) \phi\left(\mathbf{k}_{1}, t\right) \phi\left(\mathbf{k}_{2}, t\right) \theta\left(k_{c}-\left|\mathbf{k}-\mathbf{k}_{1}-\mathbf{k}_{2}\right|\right) \\
& \quad \approx\left(\frac{\tilde{g}^{2}}{2} \int^{k_{c}} \frac{d^{3} k_{1}}{(2 \pi)^{3}}\left|\phi\left(\mathbf{k}_{1}, t\right)\right|^{2}\right) \phi(\mathbf{k}, t) \equiv \Delta m_{\mathrm{cl}}^{2} \phi(\mathbf{k}, t) .
\end{aligned}
$$


Neglecting the fluctuations let us write the average influence of the other soft modes as a mass term induced by the mean field. This mass term corresponds to the lowest order tadpole contribution in the quantum field theory. Noticing the identification (C3), we find that $\Delta m_{\mathrm{cl}}^{2}$ exactly equals the expression (4) stated in the Introduction. Now, writing

$$
\dot{\phi}\left(\mathbf{k}-\mathbf{k}_{1}+\mathbf{k}_{2}, t\right) \phi\left(\mathbf{k}_{1}, t\right) \phi^{*}\left(\mathbf{k}_{2}, t\right)=\dot{\phi}(\mathbf{k}, t)\left\langle\phi\left(\mathbf{k}_{1}, t\right) \phi^{*}\left(\mathbf{k}_{2}, t\right)\right\rangle+\text { fluctuations },
$$

the first term contributes to (C1) as:

$$
\begin{aligned}
\dot{\phi}(\mathbf{k}, t) \frac{1}{4 E_{\mathbf{k}}} \int^{k_{c}} \frac{d^{3} k_{1}}{(2 \pi)^{3}}\left|\phi\left(\mathbf{k}_{1}, t\right)\right|^{2} & \left(i \mathcal{M}^{(d)}\left(\mathbf{k}+\mathbf{k}_{1}, E_{\mathbf{k}}+E_{k_{1}}\right)+i \mathcal{M}^{(d)}\left(\mathbf{k}+\mathbf{k}_{1}, E_{\mathbf{k}}-E_{k_{1}}\right)\right) \\
& \equiv \eta^{(d)}(\mathbf{k}, t) \dot{\phi}(\mathbf{k}, t) .
\end{aligned}
$$

With the identification (C3) this expression thus corresponds naturally to the scattering diagram obtained by cutting the 'sunset' diagram with two hard and one soft propagator as illustrated in Figure C1.

Similarly, for the dissipative contribution of $\operatorname{Re}\left(S_{\mathrm{IF}}^{(e)}\right)$ we write for the first term:

$$
\begin{aligned}
\int^{k_{c}} & \frac{d^{3} k_{1}^{\prime} d^{3} k_{2}^{\prime} d^{3} k_{1} d^{3} k_{2}}{(2 \pi)^{12}} \dot{\phi}\left(\mathbf{k}_{3}, t\right) \phi\left(\mathbf{k}_{1}, t\right) \phi\left(\mathbf{k}_{2}, t\right) \phi^{*}\left(\mathbf{k}_{1}^{\prime}, t\right) \phi^{*}\left(\mathbf{k}_{2}^{\prime}, t\right) \theta\left(k_{c}-\left|\mathbf{k}_{3}\right|\right) \\
\times & \frac{3}{8 E_{3}}\left(i \mathcal{M}^{(e)}\left(\mathbf{k}+\mathbf{k}_{1}^{\prime}+\mathbf{k}_{2}^{\prime}, E_{1}+E_{2}+E_{3}\right)+i \mathcal{M}^{(e)}\left(\mathbf{k}+\mathbf{k}_{1}^{\prime}+\mathbf{k}_{2}^{\prime},-E_{1}-E_{2}+E_{3}\right)\right. \\
& \left.+2 i \mathcal{M}^{(e)}\left(\mathbf{k}+\mathbf{k}_{1}^{\prime}+\mathbf{k}_{2}^{\prime}, E_{1}-E_{2}+E_{3}\right)\right)
\end{aligned}
$$

with $\mathbf{k}_{3}=\mathbf{k}+\mathbf{k}_{1}^{\prime}+\mathbf{k}_{2}^{\prime}-\mathbf{k}_{1}-\mathbf{k}_{2}$. The other term is of higher order in the time derivatives and can be neglected if the mode population changes weakly in time, i.e.

$$
\frac{d}{d t}|\phi(\mathbf{k}, t)|^{2} \ll E_{k}|\phi(\mathbf{k}, t)|^{2} .
$$

Again, the dominant contribution to the integral arises when $\mathbf{k}_{1} \approx \mathbf{k}_{1}^{\prime}, \mathbf{k}_{2} \approx \mathbf{k}_{2}^{\prime}$ or $\mathbf{k}_{1} \approx \mathbf{k}_{2}^{\prime}, \mathbf{k}_{2} \approx \mathbf{k}_{1}^{\prime}$, so that we approximate for a quasi-homogeneous system:

$$
\begin{aligned}
& \left\langle\phi\left(\mathbf{k}_{1}, t\right) \phi\left(\mathbf{k}_{2}, t\right) \phi^{*}\left(\mathbf{k}_{1}^{\prime}, t\right) \phi^{*}\left(\mathbf{k}_{2}^{\prime}, t\right)\right\rangle \\
\approx & (2 \pi)^{6}\left(\delta^{3}\left(\mathbf{k}_{1}-\mathbf{k}_{1}^{\prime}\right) \delta^{3}\left(\mathbf{k}_{2}-\mathbf{k}_{2}^{\prime}\right)+\delta^{3}\left(\mathbf{k}_{1}-\mathbf{k}_{2}^{\prime}\right) \delta^{3}\left(\mathbf{k}_{2}-\mathbf{k}_{1}^{\prime}\right)\right)\left|\phi\left(\mathbf{k}_{1}, t\right)\right|^{2}\left|\phi\left(\mathbf{k}_{2}, t\right)\right|^{2}
\end{aligned}
$$

Inserting this into $\mathrm{C} 8$ one obtains

$$
\begin{aligned}
& \dot{\phi}(\mathbf{k}, t) \frac{3}{4 E_{k}} \int^{k_{c}} \frac{d^{3} k_{1} d^{3} k_{2}}{(2 \pi)^{6}}\left|\phi\left(\mathbf{k}_{1}, t\right)\right|^{2}\left|\phi\left(\mathbf{k}_{2}, t\right)\right|^{2} \\
& \quad \times\left(i \mathcal{M}^{(e)}\left(\mathbf{k}+\mathbf{k}_{1}+\mathbf{k}_{2}, E_{1}+E_{2}+E_{k}\right)+i \mathcal{M}^{(e)}\left(\mathbf{k}+\mathbf{k}_{1}+\mathbf{k}_{2},-E_{1}-E_{2}+E_{k}\right)\right. \\
& \left.\quad+2 i \mathcal{M}^{(e)}\left(\mathbf{k}+\mathbf{k}_{1}+\mathbf{k}_{2}, E_{1}-E_{2}+E_{k}\right)\right) \\
& \equiv \eta^{(e)}(\mathbf{k}, t) \dot{\phi}(\mathbf{k}, t) .
\end{aligned}
$$

This corresponds to cutting the 'sunset' diagram with two internal soft propagators and one hard propagator giving rise to the various on-shell scattering and production processes as illustrated in Figure C2.

[1] D.Yu. Grigoriev and V. A. Rubakov, Nucl. Phys. B299, 6719 (1988); D.Yu. Grigoriev, V. A. Rubakov, and M. E. Shaposhnikov, Nucl. Phys. B326, 737 (1989).

[2] J. Ambjørn, T. Aksgaard, H. Porter, and M. Shaposhnikov, Nucl. Phys. B353, 346 (1991).

[3] J. Ambjørn and K. Farakos, Phys. Lett. B294, 248 (1992). 
[4] A. Bochkarev and P. De Forcrand, Phys. Rev. D44, 519 (1991); Phys. Rev. D47, 3476 (1993).

[5] J. Ambjørn and A. Krasnitz, Phys. Lett. B362, 97 (1995).

[6] B. Müller and A. Trayanov, Phys. Rev. Lett. 68, 3387 (1992).

[7] C. Gong, Phys. Lett. B298, 357 (1993); Phys. Rev. D49, 2642 (1994).

[8] T. S. Biró, C. Gong, and B. Müller, Phys. Rev. D52, 1260 (1995).

[9] M. Morikawa, Phys. Rev. D33, 3607 (1986).

[10] B. L. Hu, J. P. Paz, and Y. Zhang, Phys. Rev. D45, 2843 (1993); Phys. Rev. D47, 1576 (1993).

[11] E. Calzetta and B. L. Hu, Phys. Rev. D49, 6636 (1994).

[12] M. Gleiser and R. Ramos, Phys. Rev. D50, 2441 (1994).

[13] L. Kofman, A. Linde, and A. A. Starobinski, Phys. Rev. Lett. 73, 3195 (1994).

[14] D. Boyanovsky, H. J. deVega, R. Holman, D. S. Lee, and A. Singh, Phys. Rev. D51, 4419 (1995); D. Boyanovsky, M. D. Attanasio, H. J. de Vega, R. Holman, and D.-S. Lee, Phys. Rev. D52, 6805 (1995).

[15] R. H. Brandenberger and A. C. Davis, Phys. Lett. B332, 305 (1994).

[16] N. Turok, Phys. Rev. Lett. 63, 2625 (1989); D. N. Spergel, N. Turok, W. H. Press, and B. S. Ryden, Phys. Rev. D43, 1038 (1991).

[17] A. Schmid, J. Low Temp. 49, 609 (1982); A.O. Caldeira and A.J. Leggett, Physica 121A, 587 (1983).

[18] D. Bödeker, L. McLerran, and A. Smilga, Phys. Rev. D52, 4675 (1995).

[19] M. Morikawa, Phys. Rev. D42, 1027 (1990)

[20] F. Lombardo and F. D. Mazzitelli, Phys. Rev. D53, 2001 (1996).

[21] E. Braaten, Phys. Rev. Lett. 74, 2164 (1995).

[22] E. Braaten and A. Nieto, Phys. Rev. D51, 6990 (1995).

[23] T. Appelquist and R. D. Pisarski, Phys. Rev. D23, 2305 (1981).

[24] see e.g.: J. Rau and B. Müller, preprint < nucl-th/9505009 , Duke University (1995), to appear in Physics Reports.

[25] J. Schwinger, J. Math. Phys. 2, 407 (1961); L. V. Keldysh, Zh. Eksp. Teor. Fiz. 47, 1515 (1964) [Sov. Phys. JETP 20, 1018 (1964)].

[26] R. Feynman and F. Vernon, Ann. Phys. (N.Y.) 24, 118 (1963); R.P. Feynman and A.R. Hibbs, 'Quantum Mechanics and Path Integrals', McGraw-Hill Inc. (1965).

[27] Z. Su, L.-Y. Chen, X. Yu and K. Chou, Phys. Rev. B37, 9810 (1988).

[28] M. Morikawa and M. Sasaki, Phys. Lett. 165B, 59 (1985).

[29] W. H. Zurek, Phys. Rev. D26, 1862 (1982).

[30] J. J. Halliwell, Phys. Rev. D39, 2912 (1989).

[31] A. Albrecht, Phys. Rev. D46, 5504 (1992).

[32] M. Gell-Mann and J. B. Hartle, sl Phys. Rev. D47, 3345 (1993).

[33] S.R. DeGroot, W.A. van Leeuwen and Ch.G. van Weert, 'Relativistic Kinetic Theory', North-Holland-Publishing-Company (1980).

[34] H.A. Weldon, Phys. Rev. D28, 2007 (1983).

[35] R. P. Parwani, Phys. Rev. D45, 4695 (1992).

[36] S. Jeon, Phys. Rev. D52, 3591 (1995).

[37] E. Wang and U. Heinz, Phys. Rev. D53, 899 (1996).

[38] P. Arnold and O. Espinosa, Phys. Rev. D47, 3546 (1992).

[39] S.-B. Liao and M. Strickland, Phys. Rev. D52, 3653 (1995).

[40] E. Cortes, B.J. West and K. Lindenberg, J. Chem. Phys. 82, 2708 (1985).

[41] J. Cloutier and G.W. Semenoff, Phys. Rev. D44, 3218 (1991).

[42] J. P. Blaizot and E. Iancu, Phys. Rev. Lett. 72, 3317 (1994).

[43] A. D. Linde, Phys. Lett. B96, 239 (1980).

[44] D. J. Gross, R. D. Pisarski, and L. G. Yaffe, Rev. Mod. Phys. 53, 43 (1981).

[45] T. S. Biró and B. Müller, Nucl. Phys. A561, 477 (1993).

[46] P. Arnold and L. Yaffe, Phys. Rev. D52, 7208 (1995).

[47] U. Heinz, Ann. Phys. (NY) 161, 48 (1985); 168, 148 (1986).

[48] J. P. Blaizot and E. Iancu, Nucl. Phys. B417, 608 (1994).

[49] P. F. Kelly, O. Liu, C. Lucchesi, and C. Manuel, Phys. Rev. Lett. 72, 3461 (1994).

[50] S.-B. Liao, Phys. Rev. D53, 2020 (1996).

[51] E. Braaten and R. D. Pisarski, Phys. Rev. Lett. 64, 1338 (1990); Phys. Rev. D42, 2156 (1990).

[52] K. Chou, Z. Su, B. Hao and L. Yu, Phys. Rep. 118, 1 (1985).

[53] N.P. Landsman and Ch. G. van Weert, Phys. Rep. 145, 141 (1985).

[54] D. Boyanovsky, I.D. Lawrie and D.S. Lee, preprint hep-ph/9603217, University of Pittsburgh (1996). 


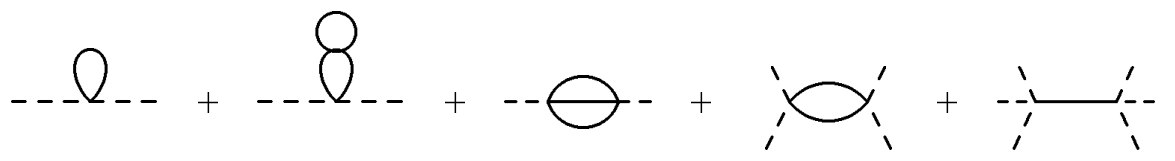

(a)

(c)

(d)

(e)

FIG.1. Feynman diagrams contributing to the influence action up to order $g^{4}$. Full lines denote hard modes and dashed lines correspond to soft modes.

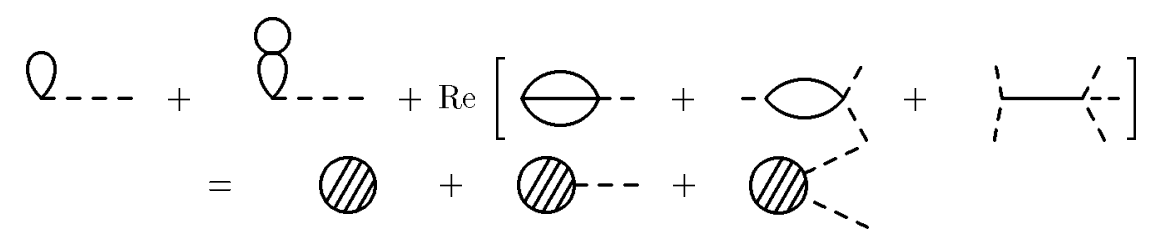

FIG.2. Graphical representation of the classical equation (29) for the soft field modes. The noise terms $\phi^{N-1} \xi_{N}$ are shown as blobs with $(N-1)$ external legs.

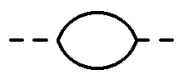

(a)
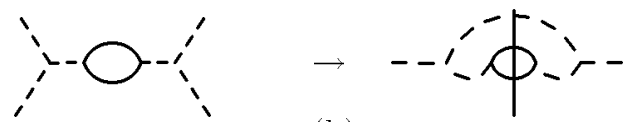

(b)

FIG.3. (a) A particular contribution to the effective action at order $g^{2}$ in $\Phi^{3}$-theory; (b) Lowest order diagram leading to on-shell dissipation when iterating the real part $\operatorname{Re}\left(S_{I F}^{(a)}\right)$ of (a) together with the $\frac{g}{3 !} \phi^{3}$ term in the full nonlinear and semiclassical equation of motion. It corresponds to the usual two loop diagram when cut through the hard thermal loop and the soft (thermal) field line. 


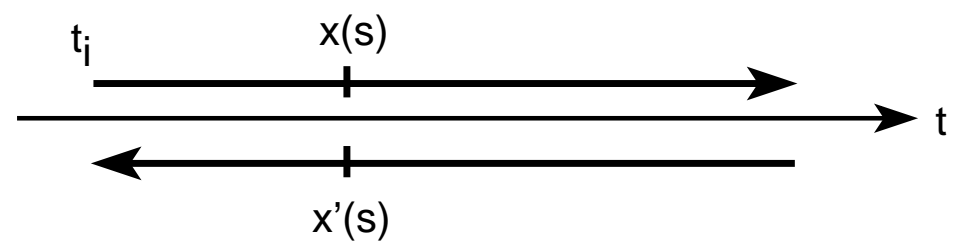

FIG.A1. Schwinger-Keldysh time contour path $\mathcal{C}$ for the variables $x(s)$ and $x^{\prime}(s)$ running from $s=t_{i}$ to $+\infty$ and back to $t_{i}$.

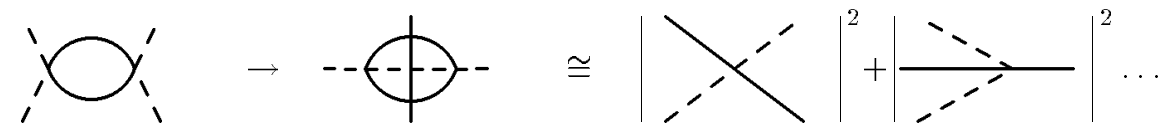

FIG.C1. Various scattering contributions when cutting the "sunset" diagramm with two hard particles and one soft mode.

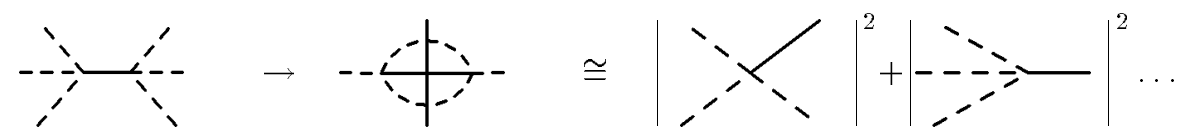

FIG.C2. Various scattering contributions when cutting the 'sunset' diagram with one hard particle and two soft modes. 